JOURNAL OF THE AMERICAN MATHEMATICAL SOCIETY

Volume 9, Number 2, April 1996

\title{
GROMOV INVARIANTS FOR HOLOMORPHIC MAPS FROM RIEMANN SURFACES TO GRASSMANNIANS
}

\author{
AARON BERTRAM, GEORGIOS DASKALOPOULOS, AND RICHARD WENTWORTH
}

Dedicated to Professor Raoul Bott on the occasion of his 70th birthday

\section{INTRODUCTION}

In [G1] Gromov introduced the space of pseudo-holomorphic curves in order to obtain new global invariants of symplectic manifolds. The theory has turned out to have far-ranging applications, perhaps the most spectacular being to the Arnold conjecture and the subsequent development of Floer homology (see $[\mathrm{F}]$ ). The Donaldson-type version of Gromov's invariants [G2] has not been much studied by mathematicians (see, however, $[R]$ ), although in the physics literature they arise naturally in the topological quantum field theories that have attracted so much attention recently (see [Wi]). In this paper we shall provide a framework for the calculation of these invariants for the case of holomorphic maps from a fixed compact Riemann surface $C$ of genus $g$ to the Grassmannian $G(r, k)$ of complex $r$-planes in $\mathbb{C}^{k}$.

To introduce some notation, let $\mathcal{M}$ (or $\mathcal{M}(d, r, k)$ ) denote the space of holomorphic maps $f: C \rightarrow G(r, k)$ of degree $d$. For simplicity we shall usually omit the data $d, r, k$, and since $C$ will always be fixed we suppress it from the notation as well. Furthermore, we will assume throughout the paper, unless otherwise indicated, that $g \geq 1$ and $d>2 r(g-1)$. The evaluation map

$$
\mu: C \times \mathcal{M} \longrightarrow G(r, k):(p, f) \mapsto f(p)
$$

defines cohomology classes on $\mathcal{M}$ by pulling back classes from $G(r, k)$ and slanting with the homology of $C$ (we will always slant with a point). Thus classes $X_{1}, \ldots, X_{l} \in H^{*}(G(r, k), \mathbb{C})$ of dimensions $n_{1}, \ldots, n_{l}$ and a point $p \in C$ define classes $\mu^{*} X_{1} / p, \ldots, \mu^{*} X_{l} / p \in H^{*}(\mathcal{M}, \mathbb{C})$, and the invariants we are interested in are the intersection numbers

$$
\left\langle X_{1}^{s_{1}} \cdots X_{l}^{s_{l}}\right\rangle=:\left(\mu^{*} X_{1} / p\right)^{s_{1}} \cup \cdots \cup\left(\mu^{*} X_{l} / p\right)^{s_{l}}[\mathcal{M}]
$$

where $\sum s_{i} n_{i}$ is the dimension of $\mathcal{M}$. As in Donaldson theory there is the usual difficulty in making sense of (1.2); one of the first problems is to find a suitable

\footnotetext{
Received by the editors June 8, 1993 and, in revised form, November 22, 1994 and March 2, 1995.

1991 Mathematics Subject Classification. Primary 14C17; Secondary 14D20, 32G13.

The first author was supported in part by NSF Grant DMS-9218215.

The second author was supported in part by NSF Grant DMS-9303494.

The third author was supported in part by NSF Mathematics Postdoctoral Fellowship DMS-
} 9007255. 
compactification of $\mathcal{M}$. This leads us to three different approaches to the problem which may roughly be characterized as the analytic, algebraic, and gauge theoretic points of view, as we shall now explain.

The analytic approach compactifies $\mathcal{M}$ through the mechanism of "bubbling" presented in the fundamental work of Sacks and Uhlenbeck [S-U]. By adding to $\mathcal{M}$ the data of a divisor on $C$ and a holomorphic map of lower degree, one obtains a compact topological space $\overline{\mathcal{M}}_{U}$ which contains $\mathcal{M}$ as an open set (in [G1] the compactness of $\overline{\mathcal{M}}_{U}$ is proved by different methods; see [Wf] for the details of applying the results of $[\mathrm{S}-\mathrm{U}]$ to this situation). We shall refer to $\overline{\mathcal{M}}_{U}$ as the Uhlenbeck compactification of $\mathcal{M}$. In $\S 4$ we shall prove

Theorem 1.3. $\overline{\mathcal{M}}_{U}(d, r, k)$ has the structure of a projective variety. For the case of maps to projective space (i.e. $r=1), \overline{\mathcal{M}}_{U}(d, 1, k)$ is smooth and is in fact a projective bundle over $J_{d}$, the Jacobian variety of degree $d$ line bundles on $C$.

Taking a cue from the theory of four manifolds, we may expect there to be an algebro-geometric compactification of $\mathcal{M}$ which perhaps contains more information than $\overline{\mathcal{M}}_{U}$. This is indeed the case, and the appropriate object is a particular case of the Grothendieck Quot Scheme, which we denote by $\overline{\mathcal{M}}_{Q}(d, r, k)$. This is a projective variety parameterizing quotients $\mathcal{O}_{C}^{k} \rightarrow \mathcal{F} \rightarrow 0$, where $\mathcal{O}_{C}$ is the structure sheaf of $C$ and $\mathcal{F}$ is a coherent sheaf on $C$ with a given Hilbert polynomial determined by $r$ and $d$. It will be seen that $\overline{\mathcal{M}}_{Q}$ naturally contains $\mathcal{M}$ as an open subvariety. The relationship with the Uhlenbeck compactification is given by the following

Theorem 1.4. There is an algebraic surjection $\overline{\mathcal{M}}_{Q}(d, r, k) \stackrel{u}{\longrightarrow} \overline{\mathcal{M}}_{U}(d, r, k)$ which is an isomorphism on $\mathcal{M}$. For the case of maps to projective space, $u$ itself is an isomorphism. Moreover, for $d$ sufficiently large (depending on $g, r$, and $k$ ) $\mathcal{M}(d, r, k)$ is dense, and the scheme structures on both $\overline{\mathcal{M}}_{Q}(d, r, k)$ and $\overline{\mathcal{M}}_{U}(d, r, k)$ are irreducible and generically reduced.

The map $u$ gives us a way to lift the calculation of intersections on $\overline{\mathcal{M}}_{U}$ to $\overline{\mathcal{M}}_{Q}$. However, in general the variety $\overline{\mathcal{M}}_{Q}$ is still singular, so it would be nice to have an explicit embedding of $\overline{\mathcal{M}}_{Q}$ in a smooth variety where the intersecting classes extended. This leads us to the third, gauge theoretic construction:

We extend Bradlow's notion of stable pairs to the case of a rank $r$ holomorphic bundle $E \rightarrow C$ with $k$ holomorphic sections $\phi_{1}, \ldots, \phi_{k}$ (cf. [B]). The definition of stability will depend on the choice of a real parameter $\tau$, and we shall see that the $(k+1)$-tuple $\left(E ; \phi_{1}, \ldots, \phi_{k}\right)$ is $\tau$-semistable if and only if it admits a solution to a certain non-linear PDE which we call the $k$ - $\tau$-vortex equation (see Theorem 3.5 ). Then following [B-D1], we construct the moduli space $\mathcal{B}_{\tau}$ of solutions. We prove

Theorem 1.5. For generic $\tau$ in a certain admissible range (see Assumption 3.12), $\mathcal{B}_{\tau}(d, r, k)$ is a smooth, projective variety. For $r=1$ and $\tau>d$,

$$
\mathcal{B}_{\tau}(d, 1, k) \simeq \overline{\mathcal{M}}_{Q}(d, 1, k) \simeq \overline{\mathcal{M}}_{U}(d, 1, k)
$$

as projective varieties.

In the process of constructing $\mathcal{B}_{\tau}$ we also verify certain universal properties and the existence of a universal rank $r$ bundle $U_{\tau} \rightarrow C \times \mathcal{B}_{\tau}$. This will allow us to prove 
Theorem 1.6. For a given $d$, there exists a choice of $\tilde{d}>d$ and a choice of $\tau$ within the range of Theorem 1.5 such that we have an algebraic embedding

$$
\overline{\mathcal{M}}_{Q}(d, r, k) \stackrel{b}{\longrightarrow} \mathcal{B}_{\tau}(\tilde{d}, r, k) .
$$

Moreover, the image $b\left(\overline{\mathcal{M}}_{Q}\right)$ is cut out by equations determined by the top Chern class of $U_{\tau}(\tilde{d}, r, k)$.

The explicit equations are the strength of Theorem 1.6; they are, of course, necessary if we want to compute intersection numbers. Other equations arise if we simply map $\overline{\mathcal{M}}_{U}(d, r, k) \rightarrow \overline{\mathcal{M}}_{U}\left(d, 1,\left(\begin{array}{l}k \\ r\end{array}\right)\right)$ via the Plücker embedding, as we shall see in $\S 4$; however, as with the Plücker embedding itself, there are too many equations, and from the point of view of computing intersections this is therefore not useful. By contrast, the equations in Theorem 1.6 cut out the Quot scheme as a complete intersection, at least for $d$ sufficiently large.

The final step in our algorithm is to extend the classes to $\mathcal{B}_{\tau}(\tilde{d}, r, k)$ and reduce the computation of intersection numbers to $\mathcal{N}(\tilde{d}, r)$, the Seshadri moduli space of rank $r$ semistable bundles on $C$ of degree $\tilde{d}$. The structure of $H^{*}(\mathcal{N}(\tilde{d}, r), \mathbb{C})$ is understood, at least in principle, from the results of Kirwan $[\mathrm{K}]$, or for $r=2$ and $\tilde{d}$ odd, from [T1] and [Z].

Actually, at present the reduction to $\mathcal{N}$ only works for $r=2$. The idea is to study the behavior of $\mathcal{B}_{\tau}$ as the parameter $\tau$ passes through a non-generic value, in a manner similar to the description given by Thaddeus in [T2] (see also [B-D-W]). The relationship is as follows: If $\tau$ is a non-generic value and $\varepsilon$ is small, then $\mathcal{B}_{\tau-\varepsilon}$ is gotten from $\mathcal{B}_{\tau+\varepsilon}$ by blowing up along a smooth subvariety and then blowing down in a different direction; a process known as a "flip". This structure arises naturally from realizing the parameter $\tau$ as the Morse function associated to a circle action on some bigger symplectic manifold and then applying the results of Guillemin and Sternberg [G-S]. In this way one eventually arrives at a value of $\tau$ for which $\mathcal{B}_{\tau}$ is a projective bundle over $\mathcal{N}$ (for even $\tilde{d}$, the fiber is only generically a projective space). One can keep track of how the intersection numbers change as $\mathcal{B}_{\tau}$ is flipped, and this in principle reduces the calculation to intersections on $\mathcal{N}$.

The computations are, of course, rather unwieldy, but to demonstrate that this procedure can actually be carried through we compute in $\S 5.3$ the case of maps from an elliptic curve to $G(2, k)$. In this case, $\operatorname{dim} \overline{\mathcal{M}}_{Q}(d, 2, k)=k d$ for $d$ large. The intersection numbers $\left\langle X_{1}^{k d-2 n} X_{2}^{n}\right\rangle, n=0,1, \ldots,[k d / 2]$ are rigorously defined in $§ 5.1$. We then prove

Theorem 1.7. For holomorphic maps of degree d sufficiently large from an elliptic curve to $G(2, k)$, the intersection numbers are given by

$$
\left\langle X_{1}^{k d-2 n} X_{2}^{n}\right\rangle=(-1)^{d+1} k 2^{k d-2 n-1}-(-1)^{d+1} \frac{k^{2}}{2} \sum_{\substack{p \in \mathbb{Z} \\
n / k \leq p \leq d-n / k}}\left(\begin{array}{c}
k d-2 n \\
k p-n
\end{array}\right) .
$$

In $\S 5.2$, we briefly discuss a remarkable conjecture concerning all the intersection numbers on $\overline{\mathcal{M}}_{U}$ which is due to Vafa and Intriligator (see [V], [I]). Using arguments from the physics of topological sigma models they derive a formula for the numbers which is based entirely on a residue calculation involving the homogeneous 
polynomial which characterizes the cohomology $\operatorname{ring} H^{*}(G(r, k), \mathbb{C})$. Somewhat surprisingly, this formula, which is simple to state yet is highly non-trivial (see Conjecture 5.10), agrees with our result Theorem 1.7 above.

As a final note, the case $r=1$, i.e. maps into projective space, has a simple description. The intersection numbers can all be computed, and we will do so in $\S 2$. It is found that these also agree with the physics formula (see Theorems 2.9 and 5.11).

\section{MAPS TO PROJECTIVE SPACE}

The purpose of this section is to compute the Gromov invariants $\left\langle X^{m}\right\rangle$ of (1.2) for the case of holomorphic maps into projective spaces. It turns out that in this case there is an obvious nonsingular compactification of the space of holomorphic maps. This can be described in terms of the push-forward of the universal line bundle on the Jacobian variety, and the computation of the intersection numbers reduces to the Poincaré formula for the theta divisor.

Let $C$ be a compact Riemann surface of genus $g$ with a base point $p \in C$. In this section only we include the case $g=0$. Let $\mathcal{M}=\mathcal{M}(d, 1, k)$ denote the space of holomorphic maps from $C$ to $\mathbb{P}^{k-1}$ of non-negative degree $d>2 g-2$. It is well-known that $\mathcal{M}$ is a complex manifold of dimension $m=k d-(k-1)(g-1)$. Let $H$ denote a fixed hyperplane in $\mathbb{P}^{k-1}$. Then we define

$$
X=\{f \in \mathcal{M}: f(p) \in H\} .
$$

Clearly, $X$ is a divisor in $\mathcal{M}$. Our goal is to compute the top intersection $\left\langle X^{m}\right\rangle$, where $m=\operatorname{dim} \mathcal{M}$ as above.

Since $\mathcal{M}$ is not compact, in order to make sense of $\left\langle X^{m}\right\rangle$ we shall define a smooth compactification $\overline{\mathcal{M}}_{P}$ of $\mathcal{M}$ and extend the class $X$ to $\overline{\mathcal{M}}_{P}$. Then we can define:

$$
\left\langle X^{m}\right\rangle=X^{m}\left[\overline{\mathcal{M}}_{P}\right] \text {, }
$$

where we denote both the extended class and its Poincaré dual by $X$, and by $\left[\overline{\mathcal{M}}_{P}\right]$ we mean the fundamental class of $\overline{\mathcal{M}}_{P}$.

Let $J_{d}$ denote the Jacobian variety of degree $d$ line bundles on $C$, and let $U \rightarrow$ $C \times J_{d}$ denote the universal or Poincaré line bundle on $C \times J_{d}$. By this we mean a line bundle whose restriction to $C \times\{L\}$ is a line bundle on $C$ isomorphic to $L$ (cf. $[\mathrm{A}-\mathrm{C}-\mathrm{G}-\mathrm{H}])$. This bundle is not uniquely determined, since we are free to tensor a given choice with any line bundle on $J_{d}$. It will be convenient to normalize $U$ so that its restriction to the point $p \in C$ :

$$
U_{p}=\left.U\right|_{\{p\} \times J_{d}}
$$

is holomorphically trivial. Let $\rho: C \times J_{d} \rightarrow J_{d}$ be the projection map. By our requirement that $d>2 g-2, \rho_{*} U$ is a vector bundle on $J_{d}$ of rank $d-g+1$. Now define

$$
\overline{\mathcal{M}}_{P}=\overline{\mathcal{M}}_{P}(d, k)=\mathbb{P}\left(\left(\rho_{*} U\right)^{\oplus k}\right),
$$

where the superscript $\oplus k$ means the fiberwise direct sum of $k$ copies of the vector bundle $\rho_{*} U$. Thus $\overline{\mathcal{M}}_{P} \stackrel{\pi}{\longrightarrow} J_{d}$ is a projective bundle with fiber over $L$ isomorphic to the projective space $\mathbb{P}\left(H^{0}(C, L)^{\oplus k}\right)$. 
Alternatively, $\overline{\mathcal{M}}_{P}$ may be thought of as gauge equivalence classes of what we shall henceforth refer to as $k$-pairs, by which we mean $(k+1)$-tuples $\left(L ; \phi_{1}, \ldots, \phi_{k}\right)$ where the $\phi_{i}$ 's are holomorphic sections of $L$ and $\vec{\phi}=:\left(\phi_{1}, \ldots, \phi_{k}\right) \not \equiv(0, \ldots, 0)$. The latter condition implies that the sections generate the fiber of $L$ at a generic point in $C$.

In order to show that $\overline{\mathcal{M}}_{P}$ forms a compactification of $\mathcal{M}$, let $\overline{\mathcal{M}}_{P}^{o} \subset \overline{\mathcal{M}}_{P}$ denote the open subvariety consisting of those $k$-pairs for which the set of $\phi_{i}$ 's generates the fiber at every point. Then we define a map

$$
F: \overline{\mathcal{M}}_{P}^{o} \longrightarrow \mathcal{M}
$$

as follows: Given a point $[L, \vec{\phi}] \in \overline{\mathcal{M}}_{P}^{o}$, let $(L, \vec{\phi})$ be a representative, and let $f: C \rightarrow \mathbb{P}^{k-1}$ be the map

$$
f(p)=\left[\phi_{1}(p), \ldots, \phi_{k}(p)\right] .
$$

Since $\vec{\phi}(p) \neq 0$ for every $p$, this is a well-defined holomorphic map which is easily seen to have degree $d$. Since a different choice of representative of $[L, \vec{\phi}]$ has the effect of rescaling each $\phi_{i}(p)$ by the same constant $\lambda(p) \in \mathbb{C}^{*}$, the map $f$ is independent of this choice, and so $F$ is well-defined and clearly holomorphic. Conversely, given $f \in \mathcal{M}$, let $S$ denote the tautological line bundle on $\mathbb{P}^{k-1}$. Then $L=f^{*} S^{*}$ is a line bundle of degree $d$ on $C$, and the coordinates of $\mathbb{C}^{k}$ pull-back to $k$ holomorphic sections $\phi_{1}, \ldots, \phi_{k}$ of $L$ generating the fiber at every point. Clearly, the map $f \mapsto[L, \vec{\phi}]$ is a holomorphic inverse of $F$. Therefore, $F$ is a biholomorphism and we have

Proposition 2.7. The projective bundle $\overline{\mathcal{M}}_{P} \stackrel{\pi}{\longrightarrow} J_{d}$ is a compactification of $\mathcal{M}$.

We will see in $\S 4$ that $\overline{\mathcal{M}}_{P}$ coincides with the Uhlenbeck compactification of $\mathcal{M}$.

Now by the top intersection of $X$ on $\mathcal{M}$ we shall mean the top intersection of its Zariski closure in $\overline{\mathcal{M}}_{P}$. Let us denote this extension and its Poincaré dual also by $X$. In order to compute the intersection number $(2.2)$ we proceed as follows: Let $\mathcal{O}_{\overline{\mathcal{M}}_{P}}(1)$ denote the anti-tautological line bundle on $\overline{\mathcal{M}}_{P}$ and $U_{p} \rightarrow J_{d}$ the restriction of $U$ to $\{p\} \times J_{d}$. Then we have

Lemma 2.8. $c_{1}\left(\mathcal{O}_{\overline{\mathcal{M}}_{P}}(1)\right)=X$.

Proof. Given a line bundle $L \rightarrow C$, consider the map

$$
\psi_{p}: H^{0}(C, L) \times \cdots \times H^{0}(C, L) \longrightarrow L_{p}:\left(\phi_{1}, \ldots, \phi_{k}\right) \longmapsto \phi_{1}(p) .
$$

Then since $U_{p}$ is trivial, $\psi_{p}$ is a well-defined linear form on the homogeneous coordinates of the fiber of $\overline{\mathcal{M}}_{P} \rightarrow J_{d}$. It follows that $s([L, \vec{\phi}])=\left(\operatorname{ker} \psi_{p}\right)^{*}$ defines a holomorphic section of $\mathcal{O}_{\overline{\mathcal{M}}_{P}}(1)$, and the zero locus of $s$ is

$$
Z(s)=\left\{[L, \vec{\phi}]: \phi_{1}(p)=0\right\} .
$$

The isomorphism $F$ in (2.5) identifies $Z(s) \cap \overline{\mathcal{M}}_{P}^{o}$ with the subspace $X$ in (2.1) (for $H$ the hyperplane defined by $\left.\left[0, z_{2}, \ldots, z_{k}\right] \in \mathbb{P}^{k-1}\right)$, and this completes the proof of the lemma.

Now we are prepared to prove the main result of this section. 
Theorem 2.9. For $X$ defined as in (2.1), non-negative $d>2 g-2$, and $m=$ $\operatorname{dim} \overline{\mathcal{M}}_{P}=k d-(k-1)(g-1)$ we have $\left\langle X^{m}\right\rangle=k^{g}$.

The computation is based on standard results on the cohomology ring of projectivized bundles. The most important tool is the notion of a Segre class; since this is perhaps not so well known to analysts, we briefly review the essentials.

Let $V$ be a rank $r$ holomorphic vector bundle on a compact, complex manifold $M$ of dimension $n$, and let $\mathbb{P}(V)$ denote the projectivization of $V$. Then $\mathbb{P}(V) \stackrel{\pi}{\longrightarrow} M$ is a projective bundle with fiber $\mathbb{P}^{r-1}$. We then have an exact sequence of bundles

$$
0 \longrightarrow \mathcal{O}_{\mathbb{P}}(-1) \longrightarrow \pi^{*} V \longrightarrow Q \longrightarrow 0
$$

where $\mathcal{O}_{\mathbb{P}}(-1)$ denotes the tautological line bundle on $\mathbb{P}(V)$ and $Q$ the quotient rank $r-1$ bundle. Let $X=c_{1}\left(\mathcal{O}_{\mathbb{P}}(1)\right)$. Then $(1-X) c(Q)=\pi^{*} c(V)$, where $c$ denotes the total Chern polynomial, or equivalently $c(Q)=\pi^{*} c(V)\left(1+X+X^{2}+\cdots\right)$. By applying the push-forward homomorphism $\pi_{*}$, or integration along the fibers, and using the fact that $\pi_{*} c_{i}(Q)=0$ for $i<r-1$ and that $c_{r-1}(Q)$ restricts to the fundamental class of the fiber, we obtain

$$
1=c(V) \pi_{*}\left(1+X+X^{2}+\cdots\right)
$$

We now define the total Segre class of $V$ by the formal expansion

$$
s(V)=\frac{1}{c(V)},
$$

and the Segre classes $s_{i}(V)$ of $V$ are defined to be the $i$-th homogeneous part of $s(V)$. It follows from (2.10) and (2.11) that for every $l \geq r-1$,

$$
\pi_{*} X^{l}=s_{l-r+1}(V)
$$

Proof of Theorem 2.9. The discussion above applies to our situation if we let $V=$ $\left(\rho_{*} U\right)^{\oplus k}$. This is a vector bundle of rank $k(d+1-g)$ on $J_{d}$, and $\overline{\mathcal{M}}_{P}=\mathbb{P}(V) \stackrel{\pi}{\longrightarrow} J_{d}$. Moreover, by Lemma 2.8 and (2.12),

$$
\left\langle X^{m}\right\rangle=X^{m}\left[\overline{\mathcal{M}}_{P}\right]=\pi_{*} X^{k(d+1-g)-1+g}\left[J_{d}\right]=s_{g}(V)\left[J_{d}\right] .
$$

It therefore suffices to compute $s_{g}(V)$. The Chern character of $V$ is computed by the Grothendieck-Riemann-Roch formula (see [A-C-G-H], p. 336)

$$
\operatorname{ch}\left(\left(\rho_{*} U\right)^{\oplus k}\right)=k \cdot \operatorname{ch}\left(\rho_{*} U\right)=k(d-g+1)-k \theta,
$$

where $\theta$ denotes the dual of the theta divisor in $J_{d}$. Moreover, as in [A-C-G-H], p. 336, the expression above implies a particularly nice form for the Chern polynomial, $c(V)=e^{-k \theta}$, and hence $s_{g}(V)=k^{g} \theta^{g} / g$ !. Applying the Poincaré formula (see [AC-G-H], p. 25) completes the proof.

It is somewhat curious that this is precisely the dimension of the space of level $k$ theta functions for genus $g$. We shall see in $§ 5.2$ that Theorem 2.9 confirms the physics conjecture for maps to projective space. 


\section{Moduli of Stable $k$-PAirs}

$\S 3.1$ Definition of stability. In this section we generalize the notion of stable pairs to stable $k$-pairs. We give the precise definition of stability for $k$-pairs and describe the associated Hermitian-Einstein equations. Since most of this section is a direct generalization of the corresponding results for stable pairs, we shall give only a brief exposition and refer to $[\mathrm{B}],[\mathrm{B}-\mathrm{D} 1,2]$ and $[\mathrm{Ti}]$ for further details.

Let $C$ be a compact Riemann surface of genus $g \geq 1$ and $E$ a complex vector bundle on $C$ of rank $r$ and degree $d>2 r(g-1)$. Unless otherwise stated, we assume that we have a fixed Kähler metric on $C$ of area $4 \pi$ and a fixed hermitian metric on $E$.

Let $\mathfrak{D}$ denote the space of $\bar{\partial}$-operators on $E$, and let $\Omega^{0}(E)$ denote the space of smooth sections of $E$. We topologize both $\mathfrak{D}$ and $\Omega^{0}(E)$ by introducing the appropriate Sobolev norms as in [B-D1]. The space of $k$-pairs is defined to be

$$
\mathcal{A}=\mathcal{A}(d, r, k)=\mathfrak{D} \times \Omega^{0}(E) \times \cdots \times \Omega^{0}(E),
$$

where we take $k$ copies of $\Omega^{0}(E)$. For example, $\mathcal{A}(d, r, 1)$ is the space of pairs considered in [B-D1]. The space of holomorphic k-pairs is defined to be

$$
\begin{gathered}
\mathcal{H}=\mathcal{H}(d, r, k)=\left\{\left(\bar{\partial}_{E}, \phi_{1}, \ldots, \phi_{k}\right) \in \mathcal{A}(d, r, k): \bar{\partial}_{E} \phi_{i}=0, i=1, \ldots, k\right. \\
\text { and } \left.\left(\phi_{1}, \ldots, \phi_{k}\right) \not \equiv(0, \ldots, 0)\right\}
\end{gathered}
$$

We shall often denote the $k$-pair $\left(\bar{\partial}_{E}, \phi_{1}, \ldots, \phi_{k}\right)$ by $\left(\bar{\partial}_{E}, \vec{\phi}\right)$. To introduce the notion of stability, define as in $[\mathrm{B}],[\mathrm{Ti}]$, the numbers

$$
\begin{gathered}
\mu_{M}(E)=\max \{\mu(F): F \subset E \text { a holomorphic subbundle with } \operatorname{rk}(F)>0\}, \\
\mu_{m}(\vec{\phi})=\min \left\{\mu\left(E / E_{\phi}\right): E_{\phi} \subset E\right. \text { a proper holomorphic subbundle with } \\
\left.\phi_{i} \in H^{0}\left(E_{\phi}\right), i=1, \ldots, k\right\} .
\end{gathered}
$$

Here $\mu$ denotes the usual Schatz slope $\mu=\operatorname{deg} / \mathrm{rk}$. Note that in the definition of $\mu_{m}(\vec{\phi})$ the set of such $E_{\phi}$ 's may be empty, in which case we set $\mu_{m}(\vec{\phi})=+\infty$. Of course, if the rank is two or greater this cannot happen with pairs, i.e. $k=1$.

Definition 3.3. A holomorphic $k$-pair $\left(\bar{\partial}_{E}, \vec{\phi}\right) \in \mathcal{H}$ is called $\tau$-stable for $\tau \in \mathbb{R}$ if

$$
\mu_{M}(E)<\tau<\mu_{m}(\vec{\phi})
$$

A $k$-pair is called stable if it is $\tau$-stable for some $\tau$.

Note that if $k=1$ this definition agrees with the one in [B]. We shall denote by $\mathcal{V}_{\tau}=\mathcal{V}_{\tau}(d, r, k)$ the subspace of $\mathcal{H}(d, r, k)$ consisting of $\tau$-stable $k$-pairs.

It is by now standard philosophy that any reasonable stability condition corresponds to the existence of special bundle metrics. These metrics satisfy the analogue 
of the Hermitian-Einstein equations, which we now describe. Given an hermitian metric $H$ on $E$ and $\phi$ a smooth section of $E$, we denote by $\phi^{*}$ the section of $E^{*}$ obtained by taking the hermitian adjoint of $\phi$. Also, given a $\bar{\partial}$-operator $\bar{\partial}_{E}$ on $E$ we denote by $F_{\bar{\partial}_{E}, H}$ the curvature of the unique hermitian connection compatible with $\bar{\partial}_{E}$ and $H$. Finally, we define the $k$ - $\tau$-vortex equation by

$$
\sqrt{-1} * F_{\bar{\partial}_{E}, H}+\frac{1}{2} \sum_{i=1}^{k} \phi_{i} \otimes \phi_{i}^{*}=\frac{\tau}{2} I .
$$

We now have the following

Theorem 3.5. Let $\left(\bar{\partial}_{E}, \vec{\phi}\right) \in \mathcal{H}$ be a holomorphic k-pair. Suppose that for a given value of the parameter $\tau$ there is an hermitian metric $H$ on $E$ such that the $k$ - $\tau$ vortex (3.4) is satisfied. Then $E$ splits holomorphically $E=E_{\phi} \oplus E_{s}$, where

(i) $E_{s}$, if nonempty, is a direct sum of stable bundles each of slope $\tau$.

(ii) $E_{\phi}$ contains the sections $\phi_{i}$ for all $i=1, \ldots, k$, and with the induced holomorphic structure from $E$ the $k$-pair $\left(E_{\phi}, \vec{\phi}\right)$ is $\tau$-stable.

Conversely, suppose that $\left(\bar{\partial}_{E}, \vec{\phi}\right)$ is $\tau$-stable. Then the $k$ - $\tau$-vortex equation has a unique solution.

Proof. See $[\mathrm{B}]$ and $[\mathrm{Ti}]$.

$\S 3.2$ Moduli of $k$-pairs and universal bundles. In this section we construct for generic values of $\tau$ within a certain range a smooth moduli space $\mathcal{B}_{\tau}=\mathcal{B}_{\tau}(d, r, k)$ of $\tau$-stable $k$-pairs on the vector bundle $E$. Furthermore, we construct a universal rank $r$ bundle $U_{\tau} \rightarrow C \times \mathcal{B}_{\tau}$ with $k$ universal sections.

We first observe that the natural complex structures on $\mathfrak{D}$ and $\Omega^{0}(E)$ induce on the space of holomorphic $k$-pairs $\mathcal{H}$ the structure of an infinite dimensional analytic variety. It was observed in [B-D-W], Corollary 2.7, that if $k=1, \mathcal{H}$ is a complex manifold. However, this fails to be true for general $k$, and for the purpose of this paper we will restrict to an open smooth part $\mathcal{H}^{*}$ of $\mathcal{H}$ defined as follows:

Definition 3.6. If $r=1$, let $\mathcal{H}^{*}=\mathcal{H}$. If $r \geq 2$, let $\mathcal{H}^{*}$ consist of those $k$-pairs $\left(\bar{\partial}_{E}, \vec{\phi}\right) \in \mathcal{H}$ satisfying

$$
\mu_{M}(E)<\frac{d-(2 g-2)}{r-1}
$$

Proposition 3.7. $\mathcal{H}^{*}$ is a smooth, complex submanifold of $\mathcal{A}$.

Proof. Let $G: \mathcal{A} \rightarrow \Omega^{0,1}(E) \times \cdots \times \Omega^{0,1}(E)$ be the map

$$
G\left(\bar{\partial}_{E}, \vec{\phi}\right)=\left(\bar{\partial}_{E} \phi_{1}, \ldots, \bar{\partial}_{E} \phi_{k}\right) \text {. }
$$

Given $\left(\bar{\partial}_{E}, \vec{\phi}\right) \in \mathcal{H}$, the derivative of $G$ at $\left(\bar{\partial}_{E}, \vec{\phi}\right)$ is given by (cf. [B-D1], (2.11))

$$
\delta G_{\left(\bar{\partial}_{E}, \vec{\phi}\right)}\left(\alpha, \eta_{1}, \ldots, \eta_{k}\right)=\left(\bar{\partial}_{E} \eta_{1}+\alpha \phi_{1}, \ldots, \bar{\partial}_{E} \eta_{k}+\alpha \phi_{k}\right) .
$$

If the cokernel of $\bar{\partial}_{E}$ vanishes for all $\left(\bar{\partial}_{E}, \vec{\phi}\right) \in \mathcal{H}^{*}$, then the restriction of $G$ to $\mathcal{H}^{*}$ has everywhere derivative of maximal rank, and Proposition 3.7 then follows from the implicit function theorem. It therefore suffices to prove the following 
Lemma 3.8. Let $E$ be a holomorphic rank $r$ vector bundle on $C$ of degree $d$, $d>2 r(g-1)$. Suppose that either $r=1$ or $r>1$ and

$$
\mu_{M}(E)<\frac{d-(2 g-2)}{r-1}
$$

Then $H^{1}(E)=0$.

Proof. The case $r=1$ is a vanishing theorem. Suppose therefore that $r>1$. Consider the Harder-Narasimhan filtration of the holomorphic bundle $E$

$$
0=E_{0} \subset E_{1} \subset \cdots \subset E_{l}=E,
$$

where $D_{j}=E_{j} / E_{j-1}$ is semistable and $\mu_{j}=\mu\left(D_{j}\right)$ satisfies $\mu_{1}>\cdots>\mu_{l}$. In particular, we have exact sequences

$$
0 \longrightarrow E_{j-1} \longrightarrow E_{j} \longrightarrow D_{j} \longrightarrow 0
$$

where $j=1, \ldots, l$. By induction, it suffices to show $H^{1}\left(D_{j}\right)=0$. Since $D_{j}$ is semistable and $\mu_{1}>\cdots>\mu_{l}$, it is enough to show that $\mu_{l}>2 g-2$ (cf. [N], p. 134). This can be proved as follows: First write

$$
d=\operatorname{deg} E=\operatorname{deg} E_{l-1}+\operatorname{deg} D_{l}
$$

By assumption,

$$
\mu\left(E_{l-1}\right) \leq \mu_{M}(E)<\frac{d-(2 g-2)}{r-1}
$$

From (3.10) and (3.11) we obtain

$$
\begin{aligned}
\operatorname{deg} D_{l} & >d-\left(r-\operatorname{rk} D_{l}\right)\left(\frac{d-(2 g-2)}{r-1}\right) \\
& =\frac{r(2 g-2)-d}{r-1}+\operatorname{rk} D_{l}\left(\frac{d-(2 g-2)}{r-1}\right) .
\end{aligned}
$$

Since $r(2 g-2)-d<0$, we obtain

$$
\operatorname{deg} D_{l}>\operatorname{rk} D_{l}\left\{\frac{r(2 g-2)-d}{r-1}+\frac{d-(2 g-2)}{r-1}\right\}=\operatorname{rk} D_{l}(2 g-2) .
$$

Thus $\mu_{l}>2 g-2$, which completes the proof of Lemma 3.8 and Proposition 3.7.

For our construction of the moduli space, we make the following

Assumption 3.12. The admissible range of $\tau$ is defined as

$$
\frac{d}{r}<\tau<\frac{d-(2 g-2)}{r-1}
$$


Definition 3.13. A value of $\tau$ is called generic if $\tau$ is not rational of the form $p / q$ where $0<q<r$.

The construction of a moduli space of $\tau$-stable $k$-pairs will only apply for $\tau$ in the admissible range; however, we shall still be interested in other values of $\tau$. Specifically, we note the following

Proposition 3.14. Given $\tau>d$, a holomorphic $k$-pair $\left(\bar{\partial}_{E}, \vec{\phi}\right)$ is $\tau$-stable if and only if the sections $\left\{\phi_{1}, \ldots, \phi_{k}\right\}$ generically generate the fiber of $E$ on $C$. In particular, if $k<r$ then the range of values of $\tau$ for which there exist $\tau$-stable $k$-pairs is bounded.

Proof. If $\left\{\phi_{1}, \ldots, \phi_{k}\right\}$ do not generically generate the fiber of $E$, then they fail to generate at every point. Hence they span a proper subbundle $E_{\phi} \neq E$. But then

$$
\mu_{m}(\vec{\phi}) \leq \mu\left(E / E_{\phi}\right) \leq \operatorname{deg}\left(E / E_{\phi}\right) \leq d<\tau,
$$

and so $\left(\bar{\partial}_{E}, \vec{\phi}\right)$ cannot be $\tau$-stable. On the other hand, if $\left\{\phi_{1}, \ldots, \phi_{k}\right\}$ generate the fiber of $E$ generically, then they cannot span a proper subbundle, and so $\mu_{m}(\vec{\phi})=$ $\infty$. Thus to prove $\tau$-stability it suffices to show that $\mu_{M}(E) \leq d$. Consider once again the filtration (3.9). For any $E^{\prime} \subset E$ we have $\mu\left(E^{\prime}\right) \leq \mu\left(E_{1}\right)=\mu_{1}$ (see $[\mathrm{N}]$, p. 162). Therefore, we need only show $\operatorname{deg} E_{1} \leq d$. We have an exact sequence

$$
0 \longrightarrow E_{l-1} \longrightarrow E \longrightarrow E / E_{l-1} \longrightarrow 0 \text {. }
$$

Now $E / E_{l-1}$ is semistable and has non-trivial sections, since the fiber of $E$ is supposed to be generated generically by $\left\{\phi_{1}, \ldots, \phi_{k}\right\}$. Thus, $\operatorname{deg} E / E_{l-1} \geq 0$. Inductively, suppose that $\operatorname{deg} E / E_{i} \geq 0$. Then we have

$$
0 \longrightarrow E_{i} / E_{i-1} \longrightarrow E / E_{i-1} \longrightarrow E / E_{i} \longrightarrow 0 \text {. }
$$

We have shown that $\mu_{l} \geq 0$, so in particular $\operatorname{deg} E_{i} / E_{i-1}>0$ for $i<l$. Hence,

$$
\operatorname{deg} E / E_{i-1}=\operatorname{deg} E / E_{i}+\operatorname{deg} E_{i} / E_{i-1},
$$

which implies $\operatorname{deg} E / E_{i-1} \geq 0$. Finally, consider

$$
0 \longrightarrow E_{1} \longrightarrow E \longrightarrow E / E_{1} \longrightarrow 0 \text {. }
$$

Then $\operatorname{deg} E_{1}=\operatorname{deg} E-\operatorname{deg} E / E_{1} \leq d$. This completes the proof.

After this digression, we are now ready to proceed with the construction of the moduli space. Recall from $\S 3.1$ the subspace $\mathcal{V}_{\tau} \subset \mathcal{H}$ of $\tau$-stable $k$-pairs. For $\tau$ admissible in the sense of Assumption 3.12, $\mathcal{V}_{\tau}$ is an open submanifold of $\mathcal{H}^{*}$ and is therefore a smooth manifold. Moreover, the actions of the complex gauge group on $\mathfrak{D}$ and $\Omega^{0}(E)$ give an action on $\mathcal{V}_{\tau}$. We define $\mathcal{B}_{\tau}=\mathcal{B}_{\tau}(d, r, k)$ to be the quotient of $\mathcal{V}_{\tau}$ by $\mathfrak{G}^{\mathbb{C}}$. Our next task is to put a complex manifold structure on $\mathcal{B}_{\tau}$. Following [B-D1], for $\left(\bar{\partial}_{E}, \vec{\phi}\right) \in \mathcal{H}$ we define the complex

$C_{\phi}^{\bar{\partial}_{E}}: \Omega^{0}($ End $E) \stackrel{d_{1}}{\longrightarrow} \Omega^{0,1}($ End $E) \oplus \Omega^{0}(E) \oplus \cdots \oplus \Omega^{0}(E) \stackrel{d_{2}}{\longrightarrow} \Omega^{0,1}(E) \oplus \cdots \oplus \Omega^{0,1}(E)$,

where

$$
\begin{aligned}
d_{1}(u) & =\left(-\bar{\partial}_{E} u, u \phi_{1}, \ldots, u \phi_{k}\right), \\
d_{2}\left(\alpha, \eta_{1}, \ldots, \eta_{k}\right) & =\left(\bar{\partial}_{E} \eta_{1}+\alpha \phi_{1}, \ldots, \bar{\partial}_{E} \eta_{k}+\alpha \phi_{k}\right) .
\end{aligned}
$$

The properties of $C_{\phi}^{\bar{\partial}_{E}}$ that we need may be summarized as follows: 
Proposition 3.16. Let $\left(\bar{\partial}_{E}, \vec{\phi}\right) \in \mathcal{H}$. Then

(i) $C_{\phi}^{\bar{\partial}_{E}}$ is an elliptic complex.

(ii) If $\left(\bar{\partial}_{E}, \vec{\phi}\right) \in \mathcal{H}^{*}$, then $H^{2}\left(C_{\phi}^{\bar{\partial}_{E}}\right)=0$.

(iii) If $\left(\bar{\partial}_{E}, \vec{\phi}\right)$ is $\tau$-stable, then $H^{0}\left(C_{\phi}^{\bar{\partial}_{E}}\right)=0$.

(iv) For $\left(\bar{\partial}_{E}, \vec{\phi}\right) \in \mathcal{H}^{*}, \chi\left(C_{\phi}^{\bar{\partial}_{E}}\right)=k d-r(k-r)(g-1)$.

A complex slice theorem as in [B-D1], $\S 3$ now proves

Proposition 3.17. For $\tau$ satisfying Assumption 3.12, $\mathcal{B}_{\tau}$ is a complex manifold of dimension $k d-r(k-r)(g-1)$. Moreover, $T_{\left[\bar{\partial}_{E}, \vec{\phi}\right]} \mathcal{B}_{\tau} \simeq H^{1}\left(C_{\phi}^{\bar{\partial}_{E}}\right)$.

We next define a Kähler structure on $\mathcal{B}_{\tau}$. Recall that $\mathfrak{D}$ and $\Omega^{0}(E)$ have natural Kähler forms $\Omega_{\mathfrak{D}}$ and $\Omega_{\Omega^{0}(E)}$, compatible with the $L^{2}$-inner products (cf. [B-D1], $\S 4)$. More precisely, let

$$
\begin{aligned}
\Omega_{\mathfrak{D}}(\alpha, \beta) & =\sqrt{-1}\left(\langle\alpha, \beta\rangle_{\mathfrak{D}}-\langle\beta, \alpha\rangle_{\mathfrak{D}}\right) \\
\Omega_{\Omega^{0}(E)}(\eta, \nu) & =\frac{\sqrt{-1}}{2}\left(\langle\eta, \nu\rangle_{\Omega^{0}(E)}-\langle\nu, \eta\rangle_{\Omega^{0}(E)}\right) .
\end{aligned}
$$

These combine to define a Kähler form

$$
\Omega=\Omega_{\mathfrak{D}}+\Omega_{\Omega^{0}(E)}+\cdots+\Omega_{\Omega^{0}(E)}
$$

on $\mathcal{A}$ which induces Kähler forms on $\mathcal{H}^{*}$ and $\mathcal{V}_{\tau}$. We will denote all these forms also by $\Omega$. Observe that the real gauge group $\mathfrak{G}$ acts on $\mathcal{H}^{*}$ preserving $\Omega$. As in [B-D1], Proposition 4.1, we find

Proposition 3.19. The map $\Psi_{\tau}: \mathcal{H}^{*} \rightarrow$ Lie $\mathfrak{G}$ defined by

$$
\Psi_{\tau}\left(\bar{\partial}_{E}, \vec{\phi}\right)=* F_{\bar{\partial}_{E}, H}-\frac{\sqrt{-1}}{2} \sum_{i=1}^{k} \phi_{i} \otimes \phi_{i}^{*}+\frac{\sqrt{-1}}{2} \tau I
$$

is an Ad-invariant moment map for the action of $\mathfrak{G}$ on the symplectic manifold $\left(\mathcal{H}^{*}, \Omega\right)$. Here, Lie $\mathfrak{G}$ denotes the Lie algebra of $\mathfrak{G}$ and is identified with its dual via the $L^{2}$-inner product.

By performing the standard infinite dimensional version of the Marsden-Weinstein reduction (cf. [B-D1], Theorem 4.5) we obtain

Theorem 3.20. For all values of $\tau$ satisfying Assumption 3.12, $\mathcal{B}_{\tau}=\mathcal{B}_{\tau}(d, r, k)$ is a Kähler manifold of dimension $k d-r(k-r)(g-1)$. Moreover, if $\tau$ is generic in the sense of Definition 3.13, then $\mathcal{B}_{\tau}$ is compact, and is in fact a non-singular projective variety.

The last statement in the theorem above is a simple generalization of the argument in [B-D-W], Theorem 4.6. We refer to $\mathcal{B}_{\tau}(d, r, k)$ as the moduli space of $\tau$-stable $k$-pairs.

As an example, let us specialize for the moment to the case $r=1$. Then $\mathcal{H}^{*}=\mathcal{H}$ (Definition 3.6) is the entire space of holomorphic pairs. Moreover, there is no stability condition and hence no $\tau$ dependence, once $\tau>d$. For this case, we therefore denote $\mathcal{B}(d, 1, k)=\mathcal{B}_{\tau}(d, r, k)$. Moreover, comparing with $\S 2$ we have 
Theorem 3.21. $\mathcal{B}(d, 1, k)=\overline{\mathcal{M}}_{P}(d, k)$ as complex manifolds. In particular, $\mathcal{B}(d, 1, k)$ is a projective variety.

Proof. To prove this, let $p r_{1}: C \times \mathfrak{D} \longrightarrow C$ be projection onto the first factor. Then on $\widetilde{U}=p r_{1}^{*}(E)$ there is a tautological complex structure which is trivial in the direction $\mathfrak{D}$ and isomorphic to $E^{\bar{\partial}_{E}}$ on the slice $C \times\left\{\bar{\partial}_{E}\right\}$. The action of $\mathfrak{G}^{\mathbb{C}}$ lifts to $\widetilde{U}$, but $\widetilde{U}$ does not descend to the quotient $C \times J_{d}$ since the constants $\mathbb{C}^{*}$ act trivially on the base and non-trivially on the fiber of $\widetilde{U}$ (cf. [A-B], $\S 9$ ). In rank one, however, the choice of a point $p \in C$ allows us to express the gauge group as a direct product

$$
\mathfrak{G}^{\mathbb{C}} \simeq \mathfrak{G}_{p}^{\mathbb{C}} \times \mathbb{C}^{*}
$$

where $\mathfrak{G}_{p}^{\mathbb{C}}=\left\{g \in \mathfrak{G}^{\mathbb{C}}: g(p)=1\right\}$. Then the quotient of $\widetilde{U}$ by $\mathfrak{G}_{p}^{\mathbb{C}}$ defines a universal bundle $U \rightarrow C \times J_{d}$. Note that the normalization (2.3) is satisfied by this choice. Let

$$
\begin{aligned}
& \tilde{\rho}: C \times \mathfrak{D} \longrightarrow \mathfrak{D}, \\
& \rho: C \times J_{d} \longrightarrow J_{d}
\end{aligned}
$$

denote the projection maps. Since we assume $d>2 g-2$, the direct images $\tilde{\rho}_{*} \widetilde{U}$ and $\rho_{*} U$ are vector bundles on $\mathfrak{D}$ and $J_{d}$, respectively. As in (2.4), we consider

$$
\begin{aligned}
\left(\tilde{\rho}_{*} \widetilde{U}\right)^{\oplus k} & =\tilde{\rho}_{*} \widetilde{U} \oplus \cdots \oplus \tilde{\rho}_{*} \widetilde{U}, \\
\left(\rho_{*} U\right)^{\oplus k} & =\rho_{*} U \oplus \cdots \oplus \rho_{*} U,
\end{aligned}
$$

where the fiberwise direct sums of vector bundles are taken $k$ times. Since $\tilde{\rho}$ is $\mathfrak{G}_{p}^{\mathbb{C}}$ equivariant, the quotient of the vector bundle $\tilde{\rho}_{*} \widetilde{U} \rightarrow \mathfrak{D}$ by $\mathfrak{G}_{p}^{\mathbb{C}}$ is isomorphic to the bundle $\rho_{*} U \rightarrow J_{d}$, hence the quotient of the projective bundle $\mathbb{P}\left(\left(\tilde{\rho}_{*} \widetilde{U}\right)^{\oplus k}\right) \rightarrow \mathfrak{D}$ by $\mathfrak{G}_{p}^{\mathbb{C}}$ is isomorphic to $\mathbb{P}\left(\left(\rho_{*} U\right)^{\oplus k}\right)=\overline{\mathcal{M}}_{P}(d, k)$. But the quotient of $\mathbb{P}\left(\left(\tilde{\rho}_{*} \widetilde{U}\right)^{\oplus k}\right) \rightarrow \mathfrak{D}$ by $\mathfrak{G}_{p}^{\mathbb{C}}$ is the same as the quotient of $\left(\tilde{\rho}_{*} \widetilde{U}\right)^{\oplus k}-\{0\}$ by $\mathfrak{G}^{\mathbb{C}}$, which by definition is the space $\mathcal{B}(d, 1, k)$.

Now assume $r \geq 2$. In the case $d / r<\tau<\mu_{+}$, where $\mu_{+}$is the smallest rational number greater than $d / r$ which can appear as the slope of a subbundle of $E$, it is easy to see that if $\left(\bar{\partial}_{E}, \vec{\phi}\right) \in \mathcal{V}_{\tau}$, then $E^{\bar{\partial}_{E}}$ is semistable (cf. [B-D1], Proposition 1.7). In this case we obtain as in [B-D2], Theorem 4.7:

Theorem 3.25. For $r \geq 2, g \geq 2$ and $d / r<\tau<\mu_{+}$, the natural map

$$
\pi: \mathcal{B}_{\tau}(d, r, k) \longrightarrow \mathcal{N}(d, r)
$$

is a morphism of algebraic varieties, where $\mathcal{N}(d, r)$ denotes the Seshadri compactification of the moduli space of rank $r$ stable bundles of degree $d$.

In the case where $d$ and $r$ are coprime, there exists a universal bundle $V \rightarrow$ $C \times \mathcal{N}(d, r)$ such that $V$ restricted to $C \times\left\{\bar{\partial}_{E}\right\}$ is a stable bundle of degree $d$ isomorphic to $E^{\bar{\partial}_{E}}$. Let $\rho: C \times \mathcal{N} \rightarrow \mathcal{N}$ be the projection map. For $d>2 r(g-1)$, the range we are considering, the push-forward $\rho_{*} V$ is a vector bundle on $\mathcal{N}$. The map $\pi$ in Proposition 3.25 suggests the following analogue of Theorem 3.21: 
Theorem 3.26. For $r \geq 2, d / r<\tau<\mu_{+}$and $d$, $r$ coprime, $\mathcal{B}_{\tau}(d, r, k) \simeq$ $\mathbb{P}\left(\left(\rho_{*} V\right)^{\oplus k}\right)$ as projective varieties.

Proof. The proof is similar to that of Theorem 3.21, only now the lack of a decomposition (3.22) makes the construction of a universal bundle more delicate. Indeed, if $d, r$ are not coprime, such a bundle does not exist. Let $\widetilde{U} \rightarrow C \times \mathfrak{D}_{s}$ be the holomorphic bundle defined as before, where now $\mathfrak{D}_{s}$ denotes the stable holomorphic structures on $E \rightarrow C$. Since $\mathfrak{G}^{\mathbb{C}}$ does not act freely on $\mathfrak{D}_{s}, \widetilde{U}$ does not descend. However, according to [A-B], pp. 579-580, when $d$ and $r$ are coprime we can find a line bundle $\widetilde{\mathcal{L}} \rightarrow \mathfrak{D}_{s}$ with an action of $\mathfrak{G}^{\mathbb{C}}$ on which $\mathbb{C}^{*} \subset \mathfrak{G}^{\mathbb{C}}$ acts by multiplication on the fiber. Let $\tilde{\rho}$ be as in (3.23) and set

$$
\widetilde{V}=\widetilde{U} \otimes \tilde{\rho}\left(\mathcal{L}^{*}\right)
$$

Since $\mathbb{C}^{*}$ acts trivially on $\widetilde{V}$, we have an action by $\overline{\mathfrak{G}}^{\mathbb{C}}=\mathfrak{G}^{\mathbb{C}} / \mathbb{C}^{*}$, and therefore $\widetilde{V}$ descends to a universal bundle $V \rightarrow C \times \mathcal{N}$. Then clearly

$$
\tilde{\rho}_{*} \widetilde{V}=\tilde{\rho}_{*} \widetilde{U} \otimes \widetilde{\mathcal{L}}^{*},
$$

so $\mathbb{P}\left(\left(\tilde{\rho}_{*} \widetilde{V}\right)^{\oplus k}\right) \simeq \mathbb{P}\left(\left(\tilde{\rho}_{*} \widetilde{U}\right)^{\oplus k}\right)$ as projective bundles on $\mathfrak{D}_{s}$. As in the proof of Theorem 3.21, the quotient of $\mathbb{P}\left(\left(\tilde{\rho}_{*} \widetilde{V}\right)^{\oplus k}\right)$ by $\overline{\mathfrak{G}}^{\mathbb{C}}$ gives $\mathbb{P}\left(\left(\rho_{*} V\right)^{\oplus k}\right) \rightarrow \mathcal{N}(d, r)$, whereas the quotient of $\mathbb{P}\left(\left(\tilde{\rho}_{*} \widetilde{U}\right)^{\oplus k}\right)$ is by definition the space $\mathcal{B}_{\tau}(d, r, k)$. This completes the proof.

Next, we would like to show that the spaces $\mathcal{B}_{\tau}$ are fine moduli spaces parameterizing $\tau$-stable $k$-pairs. What is needed is a construction of universal bundles $U_{\tau}$ on $C \times \mathcal{B}_{\tau}$ and $k$ universal sections, i.e. a map of sheaves $\mathcal{O}_{C \times \mathcal{B}_{\tau}}^{k} \rightarrow U_{\tau}$. Let

$$
p r_{1}: C \times \mathfrak{D} \times \Omega^{0}(E) \times \cdots \times \Omega^{0}(E) \longrightarrow C
$$

denote projection onto the first factor. As before, on $p r_{1}^{*}(E)$ there is a tautological complex structure which is trivial in the direction $\mathfrak{D} \times \Omega^{0}(E) \times \cdots \times \Omega^{0}(E)$ and isomorphic to $E^{\bar{\partial}_{E}}$ on the slice $C \times\left(\bar{\partial}_{E}, \vec{\phi}\right)$. Let $\widetilde{U}_{\tau}$ denote the restriction of $p_{1}^{*}(E)$ to $C \times \mathcal{V}_{\tau}$. There are $k$ tautological holomorphic sections $\widetilde{\Phi}_{1}, \ldots, \widetilde{\Phi}_{k}$ of $\widetilde{U}_{\tau}$ defined by the property that the restriction of $\widetilde{\Phi}_{i}$ to $C \times\left\{\bar{\partial}_{E}, \phi_{1}, \ldots, \phi_{k}\right\}$ is $\phi_{i}$. Next, observe that the complex gauge group $\mathfrak{G}^{\mathbb{C}}$ acts freely on $\mathcal{V}_{\tau}$ and $U_{\tau}$, and the universal sections $\widetilde{\Phi}_{i}$ are $\mathfrak{G}^{\mathbb{C}}$-equivariant with respect to this action. This implies that $\widetilde{U}_{\tau}$ and the $\widetilde{\Phi}_{i}$ 's descend to a bundle $U_{\tau} \rightarrow C \times \mathcal{B}_{\tau}$ and universal sections $\Phi_{1}, \ldots, \Phi_{k}$. We will denote all this by

$$
\vec{\Phi}: \mathcal{O}_{C \times \mathcal{B}_{\tau}}^{k} \longrightarrow U_{\tau}
$$

as mentioned above. To summarize, we have

Proposition 3.30. There exists a universal k-pair $\left(U_{\tau}(d, r, k), \vec{\Phi}\right)$ on $C \times \mathcal{B}_{\tau}(d, r, k)$, i.e. a universal rank $r$ bundle $U_{\tau}(d, r, k) \rightarrow C \times \mathcal{B}_{\tau}(d, r, k)$ with $k$ holomorphic sections $\Phi_{1}, \ldots, \Phi_{k}$.

Given Proposition 3.30, Theorems 3.21, 3.25, and 3.26 could also be obtained by appealing directly to the universal property of $\mathcal{B}_{\tau}$. For the purposes of this paper, 
it is useful to exhibit the explicit descent of the universal bundles from the infinite dimensional spaces.

Before closing this subsection, it will be important to have some compatibility between the universal pair given in Proposition 3.30 and the universal bundle $V \rightarrow$ $C \times \mathcal{N}(d, r)$ in the case where $d$ and $r$ are coprime and $d / r<\tau<\mu_{+}$. To do this, we first give an explicit description of the anti-tautological line bundle $\mathcal{O}(1)$ on $\mathcal{B}_{\tau}(d, r, k)$ coming from the identification (3.26). Let $\widetilde{U}, \widetilde{\mathcal{L}}$, and $\widetilde{V}$ be as above. Consider the map $\tilde{\pi}:\left(\tilde{\rho}_{*} \widetilde{U}\right)^{\oplus k} \rightarrow \mathfrak{D}_{s}$. Then the lift $\tilde{\pi}^{*} \widetilde{\mathcal{L}}$ has a $\mathfrak{G}^{\mathbb{C}}$ action, and therefore the quotient defines a line bundle $\mathcal{L} \rightarrow \mathcal{B}_{\tau}$.

Proposition 3.31. The bundle $\mathcal{L} \rightarrow \mathcal{B}_{\tau}$ defined above is isomorphic to the antitautological line bundle $\mathcal{O}(1)$ under the identification (3.26).

Proof. It suffices to check that the direct image $\pi_{*} \mathcal{L} \simeq\left(\left(\rho_{*} V\right)^{\oplus k}\right)^{*}$. Since $\tilde{\pi}$ is $\overline{\mathfrak{G}}^{\mathbb{C}}$ equivariant, it suffices to check this on $\mathfrak{D}_{s}$; that is, if $\tilde{\pi}^{*} \widetilde{\mathcal{L}} / \mathbb{C}^{*}$ denotes the quotient of $\tilde{\pi}^{*} \widetilde{\mathcal{L}}$ on $\tilde{\pi}: \mathbb{P}\left(\left(\tilde{\rho}_{*} \widetilde{U}\right)^{\oplus k}\right) \rightarrow \mathfrak{D}_{s}$ (here we have used $\tilde{\pi}$ to also denote the induced map), we must show that

$$
\tilde{\pi}_{*}\left(\tilde{\pi}^{*} \widetilde{\mathcal{L}} / \mathbb{C}^{*}\right) \simeq\left(\left(\tilde{\rho}_{*} \widetilde{V}\right)^{\oplus k}\right)^{*}
$$

as $\widetilde{\mathfrak{G}}^{\mathbb{C}}$ bundles. Suppose that $\left(\tilde{\rho}_{*} \widetilde{U}\right)^{\oplus k}$ has rank $N$. Then with respect to a local trivialization, local sections of $\tilde{\pi}^{*} \widetilde{\mathcal{L}} \rightarrow\left(\tilde{\rho}_{*} \widetilde{U}\right)^{\oplus k}$ are functions on $\mathbb{C}^{N}$ with values in the sheaf of local sections of $\widetilde{\mathcal{L}}$. Requiring $\mathbb{C}^{*}$ equivariance implies that these maps are linear. Finally, pushing forward by $\tilde{\pi}$ we get an isomorphism of sheaves

$$
\tilde{\pi}_{*}\left(\tilde{\pi}^{*} \widetilde{\mathcal{L}} / \mathbb{C}^{*}\right) \simeq\left(\left(\tilde{\rho}_{*} \widetilde{U}\right)^{\oplus k}\right)^{*} \otimes \widetilde{\mathcal{L}}
$$

The result then follows from (3.28).

Now consider the universal bundle $V \rightarrow C \times \mathcal{N}(d, r)$ defined by $\widetilde{\mathcal{L}}$ as in the proof of Theorem 3.26. We have the diagram:

$$
\begin{array}{ccc}
f^{*} V & \longrightarrow & C \times\left(\rho_{*} V\right)^{\oplus k} \\
& & \downarrow f \\
V & & \\
& & \times \mathcal{N}
\end{array}
$$

where $f$ is the identity on the first factor and the bundle projection on the second. The action of $\mathbb{C}^{*}$ on $\left(\rho_{*} V\right)^{\oplus k}$ lifts to $f^{*} V$. Then it follows exactly as in the proof of Proposition 3.31 that the quotient bundle $f^{*} V / \mathbb{C}^{*}$ on $C \times \mathbb{P}\left(\left(\rho_{*} V\right)^{\oplus k}\right)$ is isomorphic to $\bar{f}^{*} V \otimes \mathcal{O}(1)$, where $\bar{f}$ denotes the map $C \times \mathbb{P}\left(\left(\rho_{*} V\right)^{\oplus k}\right) \rightarrow C \times \mathcal{N}$ induced by $f$. Moreover, observe that the tautological sections of $f^{*} V \rightarrow C \times\left(\rho_{*} V\right)^{\oplus k}$ are invariant under the action of $\mathbb{C}^{*}$, and so we obtain universal sections $\vec{\Psi}: \mathcal{O}_{C \times \mathbb{P}\left(\left(\rho_{*} V\right)^{\oplus k}\right)}^{k} \rightarrow$ $\bar{f}^{*} V \otimes \mathcal{O}(1)$.

Corollary 3.32. In the case where $d / r<\tau<\mu_{+}$and $d$ and $r$ are coprime, the identification (3.26) gives an isomorphism of the $k$-pairs $\left(U_{\tau}(d, r, k), \vec{\Phi}\right)$ and $\left(\bar{f}^{*} V \otimes \mathcal{O}(1), \vec{\Psi}\right)$.

Proof. The isomorphism of bundles follows from the definition of $U_{\tau},(3.27)$, and Proposition 3.31. The fact that the sections pull back is straightforward to verify. 
$\S 3.3$ Masterspace and flips. In this subsection we specialize to the case $r=2$ and examine the dependence of the moduli spaces $\mathcal{B}_{\tau}$ on the parameter $\tau$ within the admissible range $(d / 2, d-(2 g-2))$. More precisely, we show as in [B-D-W] that when we pass an integer value in $(d / 2, d-(2 g-2))$, i.e. the non-generic values in the sense of Definition 3.13, the spaces $\mathcal{B}_{\tau}$ are related by a flip, by which we mean simply a blow-up followed by a blow-down of the exceptional divisor in a different direction. We show this by directly generalizing the "master space" construction developed in [B-D-W]. Since this part is quite straightforward we shall be brief and refer to the latter paper for more details. Finally, we describe the relationship between the universal bundles $U_{\tau}$ on the various $\mathcal{B}_{\tau}$ 's. This will be important for the calculations in $\S 5.3$.

Recall that the space $\mathcal{H}^{*}$ of rank two holomorphic $k$-pairs $\left(\bar{\partial}_{E}, \vec{\phi}\right)$ satisfying $\mu_{M}(E)<d-(2 g-2)$ has the structure of a Kähler manifold and is acted on holomorphically and symplectically by the gauge group $\mathfrak{G}$. We may define a character on $\mathfrak{G}$ as follows (cf. [B-D-W], §2): First, choose a splitting $\mathfrak{G} \simeq \mathfrak{G}_{1} \times \Upsilon$, where $\Upsilon$ is the group of components of $\mathfrak{G}$ and $\mathfrak{G}_{1}$ denotes the connected component of the identity. For $g \in \mathfrak{G}_{1}$ we define $\chi_{1}(g)$ to be the unique element of $\mathrm{U}(1)$ such that $\operatorname{det} g=\chi_{1}(g) \exp u$, where $u: C \rightarrow i \mathbb{R}$ satisfies $\int_{C} u=0$. We extend to a character $\chi$ on $\mathfrak{G}$ by letting $\chi_{1}$ act trivially on $\Upsilon$. Let $\mathfrak{G}_{0}$ denote the kernel of $\chi$, i.e. we have an exact sequence of groups

$$
1 \longrightarrow \mathfrak{G}_{0} \longrightarrow \mathfrak{G} \stackrel{\chi}{\longrightarrow} \mathrm{U}(1) \longrightarrow 1
$$

The new space $\widehat{\mathcal{B}}$ is then obtained by symplectic reduction of $\mathcal{H}^{*}$ by the action of the smaller group $\mathfrak{G}_{0}$. Specifically, by Proposition 3.19,

$$
\Psi\left(\bar{\partial}_{E}, \vec{\phi}\right)=* F_{\bar{\partial}_{E}, H}-\frac{\sqrt{-1}}{2} \sum_{i=1}^{k} \phi_{i} \otimes \phi_{i}^{*}
$$

is a moment map for the action of $\mathfrak{G}$ on $\mathcal{H}^{*}$. Hence, as in [B-D-W], Proposition 2.4

$$
\Psi_{0}\left(\bar{\partial}_{E}, \vec{\phi}\right)=\Psi\left(\bar{\partial}_{E}, \vec{\phi}\right)-\frac{1}{2} \int_{C} \operatorname{tr} \Psi\left(\bar{\partial}_{E}, \vec{\phi}\right) \cdot I
$$

is a moment map for the action of $\mathfrak{G}_{0}$. Since $r=2$, one can show that $\mathfrak{G}_{0}$ and its complexification $\mathfrak{G}_{0}^{\mathbb{C}}$ act freely on $\mathcal{H}^{*}$ (cf. [B-D-W], Proposition 2.15). It then follows that

$$
\widehat{\mathcal{B}}=\Psi_{0}^{-1}(0) / \mathfrak{G}_{0}
$$

is a smooth, symplectic manifold. A small variation of Proposition 3.20 along the lines of $[\mathrm{B}-\mathrm{D}-\mathrm{W}]$, Theorem 2.12 then proves that $\widehat{\mathcal{B}}$ is a complex manifold with the symplectic structure giving $\widehat{\mathcal{B}}$ a Kähler manifold structure. Finally, observe as in [B-D-W], Proposition 2.13, that there is a residual $\mathfrak{G} / \mathfrak{G}_{0} \simeq \mathrm{U}(1)$ action on $\widehat{\mathcal{B}}$ that is quasifree (i.e. the stabilizer of every point is either trivial or the whole $\mathrm{U}(1)$ ), holomorphic, and symplectic. Let $f: \widehat{\mathcal{B}} \rightarrow \mathbb{R}$ denote the associated moment map. As in [B-D-W], Proposition 2.13, it follows that $f$ is given by

$$
f\left(\bar{\partial}_{E}, \vec{\phi}\right)=\frac{1}{8 \pi} \sum_{i=1}^{k}\left\|\phi_{i}\right\|_{L^{2}}^{2}+\frac{d}{2} .
$$


The generic (i.e. non-integral) values of $\tau \in(d / 2, d-(2 g-2))$ correspond to the level sets where the $\mathrm{U}(1)$ action is free, hence the reduced spaces $f^{-1}(\tau) / \mathrm{U}(1)$ for such $\tau$ inherit a Kähler manifold structure, and indeed $\mathcal{B}_{\tau} \simeq f^{-1}(\tau) / \mathrm{U}(1)$ as Kähler manifolds. We summarize the above discussion by the following

Theorem 3.38. There is a Kähler manifold $\widehat{\mathcal{B}}$ with a holomorphic, symplectic, quasifree $\mathrm{U}(1)$ action whose associated moment map is given by (3.37). Moreover, with the induced Kähler structure from $\widehat{\mathcal{B}}, f^{-1}(\tau) / \mathrm{U}(1) \simeq \mathcal{B}_{\tau}$ as Kähler manifolds for any non-integer value $\tau \in(d / 2, d-(2 g-2))$.

Note that the tangent space of $\widehat{\mathcal{B}}$ at a point $[\bar{\partial}, \vec{\phi}]$ is canonically identified with $H^{1}$ of the following complex which we denote by $C_{\phi, 0}^{\widehat{\partial}_{E}}$ :

$\Omega^{0}(\text { End } E)_{0} \stackrel{d_{1}}{\longrightarrow} \Omega^{0,1}($ End $E) \oplus \Omega^{0}(E) \oplus \cdots \oplus \Omega^{0}(E) \stackrel{d_{2}}{\longrightarrow} \Omega^{0,1}(E) \oplus \cdots \oplus \Omega^{0,1}(E)$,

where $d_{1}$ and $d_{2}$ are as in (3.15) and

$$
\Omega^{0}(\text { End } E)_{0}=\left\{u \in \Omega^{0}(\text { End } E): \int_{C} \operatorname{tr} u=0\right\}
$$

The space $\widehat{\mathcal{B}}$ is the key to understanding the relationship between the $\mathcal{B}_{\tau}$ 's for different values of $\tau$. As explained in [B-D-W], this picture may best be understood via the Morse theory of the function $f$ on $\widehat{\mathcal{B}}$. First, observe that the critical values of $f$ are precisely the non-generic values of $\tau$, i.e. the integers in $(d / 2, d-(2 g-2))$. For generic $\tau<\tau^{\prime}$, if $\left[\tau, \tau^{\prime}\right] \cap \mathbb{Z}=\emptyset$, then the gradient flow of $f$ gives a biholomorphism $\mathcal{B}_{\tau} \simeq \mathcal{B}_{\tau^{\prime}}$, and indeed by Definition $3.3, \mathcal{B}_{\tau}=\mathcal{B}_{\tau^{\prime}}$ as complex manifolds (though not as Kähler manifolds). Next, suppose that $\tau$ is a critical value of $f$, and let $Z_{\tau}$ denote the critical set corresponding to $\tau$. As in [B-D-W], Example 2.21, it follows from Theorem 3.5 that

$$
Z_{\tau} \simeq \mathcal{B}(d-\tau, 1, k) \times J_{\tau}
$$

where as in $\S 2, J_{\tau}$ denotes the degree $\tau$ Jacobian variety of $C$. Let $\widehat{\mathcal{W}}_{\tau}^{+}\left(\widehat{\mathcal{W}}_{\tau}^{-}\right)$ denote the stable (unstable) manifolds of gradient flow by the function $f$. We can express $\widehat{\mathcal{W}}_{\tau}^{ \pm}$in terms of extensions of line bundles as follows (cf. [B-D-W], $\S 3$ and $\S 4.1)$ :

Proposition 3.40. (i) $\widehat{\mathcal{W}}_{\tau}^{+}$consists of equivalence classes of stable $k$-pairs $(E, \vec{\phi})$ such that $E$ fits into an exact sequence

$$
0 \longrightarrow F \longrightarrow E \stackrel{\pi}{\longrightarrow} Q_{\phi} \longrightarrow 0
$$

under the isomorphism (3.39), $\left[\left(Q_{\phi}, \pi(\vec{\phi})\right) \oplus F\right] \in Z_{\tau}$, and $\tau$ is maximal with respect to this property. Moreover, the gradient flow of $f$ takes the class of $(E, \vec{\phi})$ to the class of $\left(Q_{\phi}, \pi(\vec{\phi})\right) \oplus F$;

(ii) $\widehat{\mathcal{W}}_{\tau}^{-}$consists of equivalence classes of stable $k$-pairs $(E, \vec{\phi})$ such that $E$ fits into an exact sequence

$$
0 \longrightarrow E_{\phi} \longrightarrow E \longrightarrow F \longrightarrow 0
$$


under the isomorphism (3.39), $\left[\left(E_{\phi}, \vec{\phi}\right) \oplus F\right] \in Z_{\tau}$, and $\tau$ is minimal with respect to this property. Moreover, the inverse gradient flow of $f$ takes the class of $(E, \vec{\phi})$ to the class of $\left(E_{\phi}, \vec{\phi}\right) \oplus F$.

Proof. Immediate generalization of [B-D-W], Propositions 3.1 and 3.3.

Pick $\tau \in(d / 2, d-(2 g-2)) \cap \mathbb{Z}$. Let $W_{\tau}^{ \pm} \rightarrow Z_{\tau}$ denote the tangent bundles $T_{Z_{\tau}} \widehat{\mathcal{W}}_{\tau}^{ \pm}$to the stable and unstable strata along $Z_{\tau}$. From the complex $C_{\phi, 0}^{\bar{\partial}_{E}}$ it follows that the fiber of $W_{\tau}^{-}$at a point $\left[\bar{\partial}_{E_{\phi}} \oplus \bar{\partial}_{E_{s}}, \vec{\phi}\right] \in Z_{\tau}$ is canonically identified with $H^{1}$ of the complex

$$
\Omega^{0}\left(E_{\phi} \otimes E_{s}^{*}\right) \stackrel{d_{0}^{-}}{\longrightarrow} \Omega^{0,1}\left(E_{\phi} \otimes E_{s}^{*}\right) \longrightarrow 0
$$

where $d_{0}^{-} u=-\bar{\partial}_{E_{\phi} \otimes E_{s}^{*}} u$. Similarly, the fiber of $W_{\tau}^{+}$is identified with $H^{1}$ of the complex

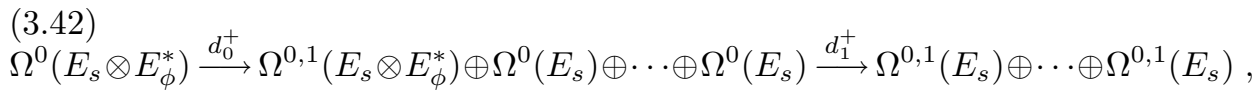

where

$$
\begin{aligned}
d_{0}^{+} u & =\left(-\bar{\partial}_{E_{s} \otimes E_{\phi}^{*}} u, u \phi_{1}, \ldots, u \phi_{2}\right), \\
d_{1}^{+}(\alpha, \vec{\psi}) & =\left(\bar{\partial}_{E_{s}} \psi_{1}+\alpha \phi_{1}, \ldots, \bar{\partial}_{E_{s}} \psi_{k}+\alpha \phi_{k}\right) .
\end{aligned}
$$

Now choose $\varepsilon>0$ sufficiently small such that $\tau$ is the only integer value in $[\tau-\varepsilon, \tau+\varepsilon]$. Let $\mathcal{W}_{\tau \pm \varepsilon}$ be the images of $\widehat{\mathcal{W}}_{\tau}^{ \pm} \cap f^{-1}(\tau \pm \varepsilon)$ under the quotient map $f^{-1}(\tau \pm \varepsilon) \rightarrow \mathcal{B}_{\tau \pm \varepsilon}=f^{-1}(\tau \pm \varepsilon) / \mathrm{U}(1)$. It follows that there are biholomorphisms $\mathcal{W}_{\tau \pm \varepsilon} \stackrel{\sim}{\longrightarrow} \mathbb{P}\left(W_{\tau}^{ \pm}\right)$making the following diagram commute:

$$
\begin{array}{ccc}
\mathcal{W}_{\tau \pm \varepsilon} & \stackrel{\sim}{\longrightarrow} & \mathbb{P}\left(W_{\tau}^{ \pm}\right) \\
\downarrow \sigma_{ \pm} & & \downarrow \\
Z_{\tau} & = & Z_{\tau}
\end{array}
$$

where the map on the right is bundle projection. Indeed, consider for example $\widehat{\mathcal{W}}_{\tau}^{+}$. Given a split pair $\left(\bar{\partial}_{E_{\phi} \oplus E_{s}}, \vec{\phi}\right)$, the map

$$
\begin{aligned}
& \operatorname{ker} d_{1}^{+} \longrightarrow \mathcal{H}^{*}, \\
& (\alpha, \vec{\psi}) \longrightarrow\left(\bar{\partial}_{E_{\phi} \oplus E_{s}}+\left(\begin{array}{cc}
0 & \alpha \\
0 & 0
\end{array}\right), \vec{\phi}+\left(\begin{array}{c}
0 \\
\vec{\psi}
\end{array}\right)\right)
\end{aligned}
$$

preserves $\mathfrak{G}_{0}^{\mathbb{C}}$ orbits and descends to give a biholomorphism $W_{\tau}^{+} \rightarrow \widehat{\mathcal{W}}_{\tau}^{+}$. By Proposition 3.40, the Morse flow $\hat{\sigma}$ corresponds to bundle projection under this equivalence, and it is easily checked that the $\mathbb{C}^{*}$ actions are compatible.

Furthermore, by direct application of the Morse theory and the description of Guillemin and Sternberg [G-S] one can show as in [B-D-W], Theorem 4.9,

Theorem 3.44. Let $\tau$ and $\varepsilon$ be as above. Then there is a smooth projective variety $\widetilde{\mathcal{B}}_{\tau}$ and holomorphic maps $p_{ \pm}$

$$
\mathcal{B}_{\tau-\varepsilon}{ }^{p_{-}} \swarrow^{\widetilde{\mathcal{B}}_{\tau}} \searrow^{p_{+}}{ }^{\mathcal{B}_{\tau+\varepsilon}}
$$

Moreover, $p_{ \pm}$are blow-down maps onto the smooth subvarieties $\mathcal{W}_{\tau \pm \varepsilon} \simeq \mathbb{P}\left(W_{\tau}^{ \pm}\right)$, and the exceptional divisor $A \subset \widetilde{\mathcal{B}}_{\tau}$ is the fiber product $A \simeq \mathbb{P}\left(W_{\tau}^{-}\right) \times_{Z_{\tau}} \mathbb{P}\left(W_{\tau}^{+}\right)$.

We end this section with some important facts concerning the universal bundles $U_{\tau}$ and the normal bundles to the centers $\mathcal{W}_{\tau \pm \varepsilon}$. 
Proposition 3.45. Let $\hat{\sigma}_{ \pm}: \widehat{\mathcal{W}}_{\tau}^{ \pm} \rightarrow Z_{\tau}$ denote the gradient flow of $f$. Then the normal bundles to the submanifolds $\widehat{\mathcal{W}}_{\tau}^{ \pm} \hookrightarrow \widehat{\mathcal{B}}$ are holomorphically isomorphic to $\hat{\sigma}_{ \pm}^{*} W_{\tau}^{\mp}$.

For the proof, we begin by giving an explicit description of the stable and unstable manifolds $\widehat{\mathcal{W}}_{\tau}^{ \pm}$. We regard $E \rightarrow C$ as a complex rank two vector bundle with a fixed hermitian metric. Let $\Omega^{0}(\mathbb{P}(E))$ denote the space of smooth sections of the associated projective bundle. Using the metric, we can identify points in $\Omega^{0}(\mathbb{P}(E))$ with smooth projections $\pi$ of rank one; hence, $\pi: E \rightarrow E, \pi^{2}=\pi^{*}=\pi$. Note that if $E$ has a holomorphic structure $\bar{\partial}_{E}$, the condition that $L=\pi E$ define a holomorphic line subbundle is $\pi^{\perp} \bar{\partial}_{E} \pi=0$, where $\pi^{\perp}=I-\pi$. Let $\mathcal{E}=\mathcal{A} \times \Omega^{0}(\mathbb{P}(E)$ ). Then $\mathcal{E}$ has the structure of a complex Fréchet manifold. In the following, we shall also implicitly make use of the usual Sobolev completions. Associated to each $\pi$ we have the degree of the line subbundle $\pi E$. Let $\mathcal{E}_{c}$ denote the component of $\mathcal{E}$ where this degree is $c$.

Now fix a critical $\tau$ as above. We let $\mathcal{E}_{+}=\mathcal{E}_{\tau}, \mathcal{E}_{-}=\mathcal{E}_{d-\tau}$. Note that we have holomorphic projections

$$
\tilde{\jmath}_{ \pm}: \mathcal{E}_{ \pm} \longrightarrow \mathcal{A}
$$

with fiber $\Omega^{0}(\mathbb{P}(E))$. Let

$$
\widetilde{\mathcal{S}}_{-}=\left\{\left(\bar{\partial}_{E}, \vec{\phi}, \pi\right) \in \mathcal{E}_{-}: \bar{\partial}_{E} \phi_{i}=0, \pi^{\perp} \phi_{i}=0, \pi^{\perp} \bar{\partial}_{E} \pi=0, i=1, \ldots, k\right\} .
$$

Note that if $\left(\bar{\partial}_{E}, \vec{\phi}, \pi\right) \in \widetilde{\mathcal{S}}_{-}$, then the holomorphic bundle $E^{\bar{\partial}_{E}}$ fits into an exact sequence

$$
0 \longrightarrow E_{\phi} \longrightarrow E \longrightarrow E_{s} \longrightarrow 0
$$

where $E_{\phi}=\pi E, \phi_{i} \in H^{0}\left(E_{\phi}\right)$ for $i=1, \ldots, k$, and $E_{s}=\pi^{\perp} E$. Also, by the choice of $\tau,\left(\bar{\partial}_{E}, \vec{\phi}\right) \in \mathcal{H}^{*}$.

Lemma 3.47. $\widetilde{\mathcal{S}}_{-}$is a $\mathfrak{G}^{\mathbb{C}}$-invariant smooth complex submanifold of $\mathcal{E}_{-}$.

Proof. The invariance is clear. For smoothness, let $V \rightarrow \mathcal{E}_{-}$be the holomorphic vector bundle whose fiber over $\left(\bar{\partial}_{E}, \vec{\phi}, \pi\right)$ is

$$
\Omega^{0,1}(E) \oplus \cdots \oplus \Omega^{0,1}(E) \oplus \Omega^{0}\left(\operatorname{Hom}\left(\pi(E), \pi^{\perp}(E)\right)\right) .
$$

We define a section

$$
\begin{gathered}
F: \mathcal{E}_{-} \longrightarrow V, \\
F\left(\bar{\partial}_{E}, \vec{\phi}, \pi\right)=\left(\bar{\partial}_{E} \vec{\phi}, \pi^{\perp} \bar{\partial}_{E} \pi\right) .
\end{gathered}
$$

Then a computation of the derivative shows that $F$ is transversal to the zero section of $V$ (cf. [D-U]). It follows that $\widetilde{\mathcal{E}}_{-}=F^{-1}$ (zero section) is a smooth submanifold of $\mathcal{E}_{-}$. Note that we have used the assumption that $\tau$ be in the admissible range. Clearly, $F$ is also holomorphic, so the submanifold is complex. Now consider the holomorphic vector bundle $\widetilde{V} \rightarrow \widetilde{\mathcal{E}}_{-}$whose fiber at a point $\left(\bar{\partial}_{E}, \vec{\phi}, \pi\right)$ is $H^{0}\left(\pi^{\perp}(E)\right)^{\oplus k}$. That this defines a vector bundle is again a consequence of the assumption on $\tau$. We define a section $\widetilde{F}: \widetilde{\mathcal{E}}_{-} \rightarrow \widetilde{V}$ by $\widetilde{F}\left(\bar{\partial}_{E}, \vec{\phi}, \pi\right)=\pi^{\perp} \vec{\phi}$. This section is also transverse to the zero section, since $H^{1}(\pi(E))=0$, and it is also holomorphic. Therefore, $\widetilde{\mathcal{S}}_{-}=\widetilde{F}^{-1}$ (zero section) is a smooth complex submanifold. 
Lemma 3.48. The group $\mathfrak{G}_{0}^{\mathbb{C}}$ acts freely on $\widetilde{\mathcal{S}}_{-}$giving $\mathcal{S}_{-}=\widetilde{\mathcal{S}}_{-} / \mathfrak{G}_{0}^{\mathbb{C}}$ the structure of a finite dimensional complex manifold. Let $\left(\bar{\partial}_{E}, \vec{\phi}, \pi\right) \in \widetilde{\mathcal{S}}_{-}$, and let $E_{\phi}=\pi E$, $E_{s}=\pi^{\perp} E$. Then the complex

$$
\begin{gathered}
\Omega^{0}(\text { End } E)_{0} \stackrel{f_{0}^{-}}{\longrightarrow} \Omega^{0,1}(\text { End } E) \oplus \Omega^{0}\left(E_{s} \otimes E_{\phi}^{*}\right) \oplus \Omega^{0}(E) \oplus \cdots \oplus \Omega^{0}(E) \\
\stackrel{f_{1}^{-}}{\longrightarrow} \Omega^{0,1}\left(E_{s} \otimes E_{\phi}^{*}\right) \oplus \Omega^{0,1}(E) \oplus \cdots \oplus \Omega^{0,1}(E) \oplus \Omega^{0}\left(E_{s}\right) \oplus \cdots \oplus \Omega^{0}\left(E_{s}\right) \\
\stackrel{f_{2}^{-}}{\longrightarrow} \Omega^{0,1}\left(E_{s}\right) \oplus \cdots \oplus \Omega^{0,1}\left(E_{s}\right)
\end{gathered}
$$

where

$$
\begin{aligned}
& f_{0}^{-}(u)=\left(-\bar{\partial}_{E} u, \pi^{\perp} u \pi, u \phi_{i}\right), \\
& f_{1}^{-}\left(\alpha, \eta, \psi_{i}\right)=\left(\bar{\partial}_{E_{s} \otimes E_{\phi}^{*}} \eta+\pi^{\perp} \alpha \pi,-\eta \phi_{i}+\pi^{\perp} \psi_{i}, \bar{\partial}_{E} \psi_{i}+\alpha \phi_{i}\right), \\
& f_{2}^{-}\left(\beta, \sigma_{i}, \gamma_{j}\right)=\left(\bar{\partial}_{E_{s}} \sigma_{i}+\beta \phi_{i}-\gamma_{i}\right) \text {, }
\end{aligned}
$$

is Fredholm with vanishing $H^{0}$ and $H^{2}$. Moreover, $H^{1}$ of the complex is canonically identified with the fiber of $T \mathcal{S}_{-}$at the point $\left[\bar{\partial}_{E}, \vec{\phi}, \pi\right]$.

Proof. The proof is standard deformation theory (cf. [Ko]) combined with the arguments in $[\mathrm{B}-\mathrm{D}-\mathrm{W}]$ and $[\mathrm{D}-\mathrm{U}]$. We omit the details.

Similarly, we define

$\widetilde{\mathcal{S}}_{+}=\left\{\left(\bar{\partial}_{E}, \vec{\varphi}, \pi\right) \in \mathcal{E}_{+}: \bar{\partial}_{E} \varphi_{i}=0, i=1, \ldots, k, \pi^{\perp} \bar{\partial}_{E} \pi=0, \pi \varphi_{i} \neq 0\right.$ for some $\left.i\right\}$.

As in the case of $\widetilde{\mathcal{S}}_{-}$, if we set $E_{s}=\pi E, E_{\phi}=\pi^{\perp} E$, then $E^{\bar{\partial}_{E}}$ fits into an exact sequence of holomorphic bundles

$$
0 \longrightarrow E_{s} \longrightarrow E \longrightarrow E_{\phi} \longrightarrow 0
$$

with $\left(\bar{\partial}_{E}, \vec{\varphi}\right) \in \mathcal{H}^{*}$. We also have as before

Lemma 3.49. $\widetilde{\mathcal{S}}_{+}$is a $\mathfrak{G}^{\mathbb{C}}$ invariant smooth complex submanifold of $\mathcal{E}_{+}$. The subgroup $\mathfrak{G}_{0}^{\mathbb{C}}$ acts freely giving $\mathcal{S}_{+}=\widetilde{\mathcal{S}}_{+} / \mathfrak{G}_{0}^{\mathbb{C}}$ the structure of a smooth complex manifold. Let $\left(\bar{\partial}_{E}, \vec{\varphi}, \pi\right) \in \widetilde{\mathcal{S}}_{+}$and set $E_{s}=\pi E, E_{\phi}=\pi^{\perp} E$. Then the complex

$$
\begin{gathered}
\Omega^{0}(\text { End } E)_{0} \stackrel{f_{0}^{+}}{\longrightarrow} \Omega^{0,1}(\text { End } E) \oplus \Omega^{0}\left(E_{\phi} \otimes E_{s}^{*}\right) \oplus \Omega^{0}(E) \oplus \cdots \oplus \Omega^{0}(E) \\
\stackrel{f_{1}^{+}}{\longrightarrow} \Omega^{0,1}\left(E_{\phi} \otimes E_{s}^{*}\right) \oplus \Omega^{0,1}(E) \oplus \cdots \oplus \Omega^{0,1}(E) \longrightarrow 0
\end{gathered}
$$

where

$$
\begin{aligned}
f_{0}^{+}(u) & =\left(-\bar{\partial}_{E} u, \pi^{\perp} u \pi, u \phi_{i}\right), \\
f_{1}^{+}\left(\alpha, \eta, \psi_{i}\right) & =\left(\bar{\partial}_{E_{\phi} \otimes E_{s}^{*}} \eta+\pi^{\perp} \alpha \pi, \bar{\partial}_{E} \psi_{i}+\alpha \phi_{i}\right),
\end{aligned}
$$

is Fredholm with vanishing $H^{0}$ and $H^{2}$. Moreover, $H^{1}$ of the complex is canonically identified with the fiber of $T \mathcal{S}_{+}$at the point $\left[\bar{\partial}_{E}, \vec{\varphi}, \pi\right]$.

With this preparation, we are now able to prove Proposition 3.45. Consider the maps $\tilde{\jmath}_{ \pm}$of (3.46) restricted to the submanifolds $\widetilde{\mathcal{S}}_{ \pm}$. These are $\mathfrak{G}_{0}^{\mathbb{C}}$-equivariant and their image is clearly in the $\mathfrak{G}_{0}^{\mathbb{C}}$ orbit of $\Psi^{-1}(0)$. Thus, we have holomorphic maps $\jmath_{ \pm}: \mathcal{S}_{ \pm} \rightarrow \hat{\mathcal{B}}$ which are evidently injective with image $\widehat{\mathcal{W}}_{\tau}^{ \pm}$. We claim that these are 
actually embeddings. Moreover, the description in Lemmas 3.48 and 3.49 gives the desired result about normal bundles. Both of these assertions follow from (3.41), (3.42) and considering the long exact sequence associated to a double complex. For $\widehat{\mathcal{W}}_{\tau}^{-}$, the double complex is

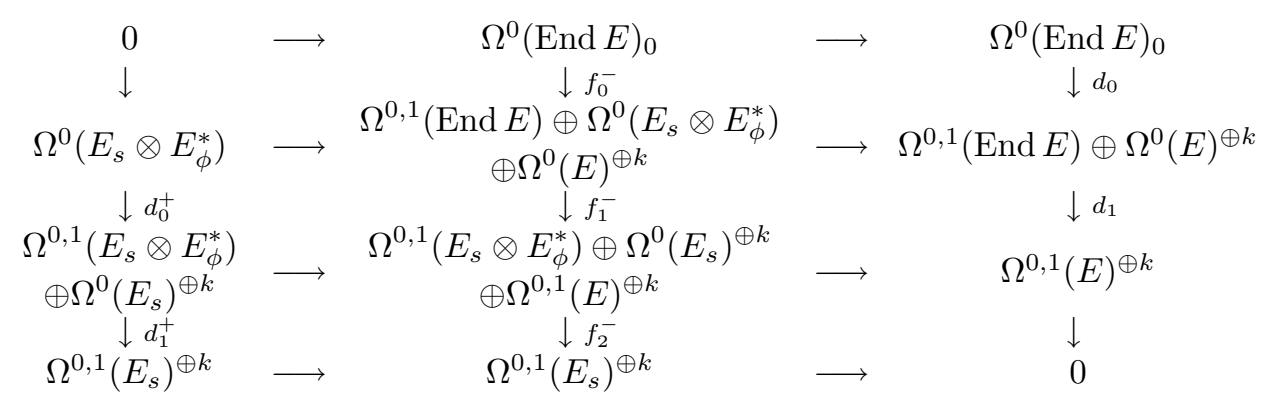

Notice that we use the vanishing of $H^{0}$ of the complex (3.42) (the left column above) which follows from the assumption $\left(\phi_{1}, \ldots, \phi_{k}\right) \neq(0, \ldots, 0)$, and the vanishing of $H^{2}$ of the complex in Lemma 3.48 (the middle column above). For $\widehat{\mathcal{W}}_{\tau}^{+}$, we have

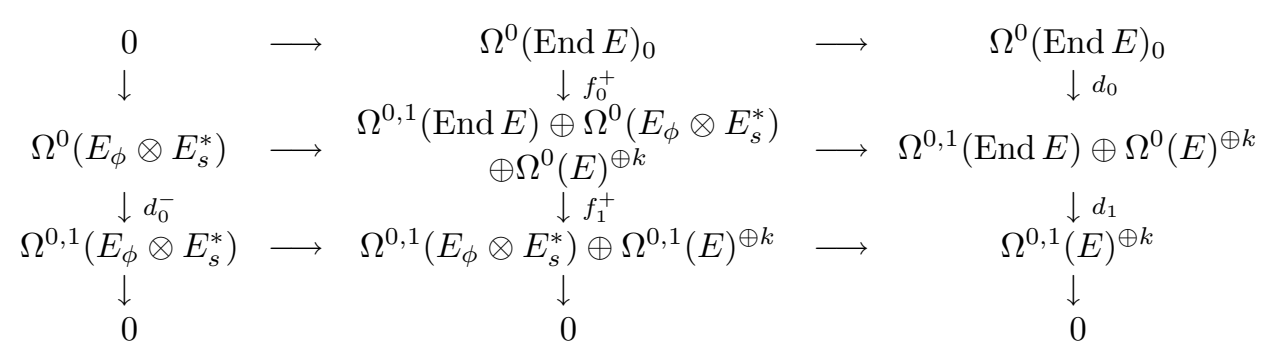

As before, $H^{0}$ of the complex in the left column above (i.e. (3.41)) vanishes since $\tau>d / 2$, and $H^{2}$ of the middle column vanishes by the assertion in Lemma 3.49.

Corollary 3.50. The normal bundle $\nu\left(\mathcal{W}_{\tau \pm \varepsilon}\right)$ of $\mathcal{W}_{\tau \pm \varepsilon}$ in $\mathcal{B}_{\tau \pm \varepsilon}$ is given by

$$
\nu\left(\mathcal{W}_{\tau \pm \varepsilon}\right)=\sigma_{ \pm}^{*}\left(W_{\tau}^{\mp}\right) \otimes \mathcal{O}_{\mathbb{P}\left(W_{\tau}^{ \pm}\right)}(-1)
$$

where $\mathcal{O}_{\mathbb{P}\left(W_{\tau}^{ \pm}\right)}(-1)$ are the tautological line bundles on $\mathcal{W}_{\tau \pm \varepsilon}$ coming from the identification (3.43).

Proof. Consider $\mathcal{W}_{\tau+\varepsilon}$ for example. By Proposition 3.45, it follows that the normal bundle to $\widehat{\mathcal{W}}_{\tau}^{+} \cap f^{-1}(\tau+\varepsilon)$ in $f^{-1}(\tau+\varepsilon)$ is the restriction of $\hat{\sigma}_{+}^{*} W_{\tau}^{-}$. Via the identification (3.43), an element $\lambda \in U(1)$ acts on $W_{\tau}^{+}$by $\lambda^{2}$ and on $W_{\tau}^{-}$by $\lambda^{-2}$. Upon taking quotients, $\hat{\sigma}_{+}^{*} W_{\tau}^{-}$therefore descends to $\sigma_{+}^{*} W_{\tau}^{-}$twisted by the tautological line bundle.

Let $\mathcal{L}_{\phi}$ and $\mathcal{L}_{s}$ denote the pullbacks to $C \times Z_{\tau}$ of the universal bundles on $C \times \mathcal{B}(d-\tau, 1, k)$ and $C \times J_{\tau}$ under the identification (3.39).

Proposition 3.51. The following are exact sequences of holomorphic bundles on $C \times \mathcal{W}_{\tau \pm \varepsilon}$ :

$$
\left.0 \longrightarrow \sigma_{-}^{*} \mathcal{L}_{\phi} \longrightarrow U_{\tau-\varepsilon}\right|_{C \times \mathbb{P}\left(W_{\tau}^{-}\right)} \longrightarrow \sigma_{-}^{*} \mathcal{L}_{s} \otimes \mathcal{O}_{\mathbb{P}\left(W_{\tau}^{-}\right)}(-1) \longrightarrow 0
$$




$$
\left.0 \longrightarrow \sigma_{+}^{*} \mathcal{L}_{s} \otimes \mathcal{O}_{\mathbb{P}\left(W_{\tau}^{+}\right)}(+1) \longrightarrow U_{\tau+\varepsilon}\right|_{C \times \mathbb{P}\left(W_{\tau}^{+}\right)} \longrightarrow \sigma_{+}^{*} \mathcal{L}_{\phi} \longrightarrow 0
$$

Proof. Recall the construction of the universal bundles $U_{\tau}$. By quotienting out by the smaller group $\mathfrak{G}_{0}^{\mathbb{C}}$ we obtain a universal bundle $\widehat{U} \rightarrow C \times \widehat{\mathcal{B}}$ in the same way. Consider $\jmath_{-}^{*} \widehat{U}$, where $\jmath_{-}$is extended to $C \times \widehat{\mathcal{W}}_{\tau}^{-}$. Then on $C \times \widehat{\mathcal{W}}_{\tau}^{-}$there is an exact sequence of holomorphic bundles

$$
0 \longrightarrow \Pi \longrightarrow \jmath_{-}^{*} \widehat{U} \longrightarrow \Pi^{\perp} \longrightarrow 0
$$

defined as follows. On $C \times \widetilde{\mathcal{S}}_{-}$consider the smooth line subbundle $\tilde{\Pi}$ whose fiber along $C \times\left\{\left(\bar{\partial}_{E}, \vec{\phi}, \pi\right)\right\}$ is the tautological line subbundle defined by $\pi$. By definition of the holomorphic structure on $\widetilde{U}$ this defines a holomorphic subbundle with quotient $\widetilde{\Pi}^{\perp}$. Since the resulting exact sequence is $\mathfrak{G}_{0}^{\mathbb{C}}$ equivariant, it descends to give (3.52).

Next we define

$$
\widetilde{Z}_{\tau}=\left\{\left(\bar{\partial}_{E}, \vec{\phi}, \pi\right) \in \widetilde{\mathcal{S}}_{-}: \pi \bar{\partial}_{E} \pi^{\perp}=0\right\}
$$

Then as in Lemma 3.47 one can show that $\widetilde{Z}_{\tau}$ is a smooth complex $\mathfrak{G}^{\mathbb{C}}$ invariant submanifold of $\widetilde{\mathcal{S}}_{-}$. Moreover, $\mathfrak{G}_{0}^{\mathbb{C}}$ acts freely and $Z_{\tau}=\widetilde{Z}_{\tau} / \mathfrak{G}_{0}^{\mathbb{C}}$. The splitting $\pi$ gives a holomorphic splitting

$$
\left.\widetilde{U}\right|_{C \times \widetilde{Z}_{\tau}}=\widetilde{\mathcal{L}}_{\phi} \oplus \widetilde{\mathcal{L}}_{s}
$$

Clearly, $\widetilde{\mathcal{L}}_{\phi}$ and $\widetilde{\mathcal{L}}_{s}$ descend under the action of $\mathfrak{G}_{0}^{\mathbb{C}}$ to universal bundles $\mathcal{L}_{\phi}$ and $\mathcal{L}_{s}$. We now claim that $\Pi=\hat{\sigma}_{-}^{*} \mathcal{L}_{\phi}$ and $\Pi^{\perp}=\hat{\sigma}_{-}^{*} \mathcal{L}_{s}$. Consider the holomorphic mapping

$$
\begin{gathered}
\widetilde{\mathcal{S}}_{-} \stackrel{p}{\longrightarrow} \widetilde{Z}_{\tau}, \\
\left(\bar{\partial}_{E}, \vec{\phi}, \pi\right) \mapsto\left(\pi \bar{\partial}_{E} \pi+\pi^{\perp} \bar{\partial}_{E} \pi^{\perp}, \vec{\phi}, \pi\right),
\end{gathered}
$$

which sends the extension to the split extension. This is not quite $\mathfrak{G}_{0}^{\mathbb{C}}$ equivariant; however, it does preserve orbits, and by Proposition 3.40 it gives a lifting of $\hat{\sigma}_{-}$. More precisely, we shall show below that for $g \in \mathfrak{G}_{0}^{\mathbb{C}}$,

$$
p\left(g\left(\bar{\partial}_{E}, \vec{\phi}, \pi\right)\right)=g h p\left(\bar{\partial}_{E}, \vec{\phi}, \pi\right),
$$

where $h \in \mathfrak{G}_{0}^{\mathbb{C}}$ depends on $g$ and $\bar{\partial}_{E}$ and has the form

$$
h=\left(\begin{array}{cc}
1 & u \\
0 & -1
\end{array}\right)
$$

with respect to the splitting $\pi$. With this understood, it is clear that $\widetilde{\Pi}=p^{*} \widetilde{\mathcal{L}}_{\phi}$; and since transformations of the form $h$ act trivially on $\widetilde{\mathcal{L}}_{\phi}$, the $\mathfrak{G}_{0}^{\mathbb{C}}$ actions are the same, and we have $\Pi=\hat{\sigma}_{-}^{*} \mathcal{L}_{\phi}$ on the quotient. Part (i) of the proposition then follows by considering the $\mathrm{U}(1)$ action as in (3.50). Part (ii) follows similarly. 
It remains to prove (3.53). Note that if $L \in \mathbb{P}(E), g$ is a complex gauge transformation, and $\pi_{K}^{L}$ denotes projection onto $L$ with respect to the metric $K$, then $\pi_{K}^{g L}=g \pi_{g K}^{L} g^{-1}$, where $g K$ is the pull-back of $K$ by $g$. On the other hand, by Gram-Schmidt we can write $\pi_{g K}^{L}=\pi_{K}^{L}+u$, where $u \in \operatorname{Hom}\left(\left(\pi_{K}^{L}\right)^{\perp}, \pi_{K}^{L}\right)$ depends on $g$. Also, we have

$$
\left(\pi_{K}^{L}\right)^{\perp} \bar{\partial}_{E} \pi_{K}^{L}=0 \Longrightarrow u \bar{\partial}_{E} \pi_{K}^{L}=0, u \bar{\partial}_{E} u=0,\left(\pi_{K}^{L}\right)^{\perp} \bar{\partial}_{E} u=0
$$

Hence,

$$
\begin{aligned}
\pi_{K}^{g L} g\left(\bar{\partial}_{E}\right) \pi_{K}^{g L} & =g\left(\pi_{K}^{L} \bar{\partial}_{E} \pi_{K}^{L}+\pi_{K}^{L} \bar{\partial}_{E} u\right) \\
\left(\pi_{K}^{g L}\right)^{\perp} g\left(\bar{\partial}_{E}\right)\left(\pi_{K}^{g L}\right)^{\perp} & =g\left(\left(\pi_{K}^{L}\right)^{\perp} \bar{\partial}_{E}\left(\pi_{K}^{L}\right)^{\perp}-u \bar{\partial}_{E}\left(\pi_{K}^{L}\right)^{\perp}\right) .
\end{aligned}
$$

Then (3.53) easily follows.

\section{Maps to Grassmannians}

$\S 4.1$ The Uhlenbeck compactification. The main purpose of this section is to show that the Uhlenbeck compactification of $\mathcal{M}(d, r, k)$, the space of holomorphic maps of degree $d$ from $C$ to $G(r, k)$, admits the structure of a projective variety. In the case of maps into projective space, it is actually a smooth, holomorphic projective bundle over the Jacobian.

We first introduce $\overline{\mathcal{M}}_{U}(d, r, k)$ set theoretically. Let $0 \leq l \leq d$ be an integer, and let $C_{l}$ denote the $l$-th symmetric product of the curve $C$. We set

$$
\overline{\mathcal{M}}_{U}(d, r, k)=\bigsqcup_{0 \leq l \leq d} C_{l} \times \mathcal{M}(d-l, r, k),
$$

and we will denote elements of $\overline{\mathcal{M}}_{U}(d, r, k)$ by ordered pairs $(D, f)$. Given $(D, f)$ we associate the distribution $e(f)+\delta_{D}$, where $e(f)=|d f|^{2}$ is the energy density of the map $f$ with respect to the fixed Kähler metrics on $C$ and $G(r, k)$, and $\delta_{D}$ is the Dirac distribution supported on $D$. We topologize $\overline{\mathcal{M}}_{U}(d, r, k)$ by giving a local basis of neighborhoods around each point $(D, f)$ as follows: Pick a basis of neighborhoods $N$ of $f \in \mathcal{M}(d-l, r, k)$ in the $C_{0}^{\infty}(C \backslash D)$ topology and a basis of neighborhoods $W$ of $e(f)+\delta_{D}$ in the weak ${ }^{*}$ topology. Set

$$
V(D, f)=\left\{\left(D^{\prime}, f^{\prime}\right) \in \overline{\mathcal{M}}_{U}(d, r, k): f^{\prime} \in N \text { and } e\left(f^{\prime}\right)+\delta_{D^{\prime}} \in W\right\} .
$$

Since both the $C_{0}^{\infty}$ and weak* topologies are first countable, so is the topology defined on $\overline{\mathcal{M}}_{U}(d, r, k)$. In terms of sequences then, $\left(D_{i}, f_{i}\right) \rightarrow(D, f)$ in $\overline{\mathcal{M}}_{U}(d, r, k)$ if and only if:

(i) $f_{i} \rightarrow f$ in the $C_{0}^{\infty}(C \backslash D)$ topology, and

(ii) $e\left(f_{i}\right)+\delta_{D_{i}} \rightarrow e(f)+\delta_{D}$ in the weak* topology.

By the theorem of Sacks and Uhlenbeck $[\mathrm{S}-\mathrm{U}], \overline{\mathcal{M}}_{U}(d, r, k)$ with this topology is compact.

Definition 4.3. The space $\overline{\mathcal{M}}_{U}(d, r, k)$ with the topology described above is called the Uhlenbeck compactification of $\mathcal{M}(d, r, k)$.

Strictly speaking, the Uhlenbeck compactification as defined above may not be a topological compactification of the space of holomorphic maps. However, provided 
$d$ is sufficiently large, $\mathcal{M}(d, r, k)$ is indeed dense in $\overline{\mathcal{M}}_{U}(d, r, k)$ (see Corollary 4.29 below).

Let us consider for the moment the case $r=1$. Then the Uhlenbeck compactification of holomorphic maps of degree $d$ from $C$ to $\mathbb{P}^{k-1}$ is defined once we have chosen Kähler metrics on $C$ and $\mathbb{P}^{k-1}$. For $C$ we use the metric of $\S 3$, and for $\mathbb{P}^{k-1}$ we choose the Fubini-Study metric. Then we have

Theorem 4.4. If $r=1$, then $\overline{\mathcal{M}}_{U}(d, 1, k)$ is homeomorphic to $\mathcal{B}(d, 1, k)$. In particular, the Uhlenbeck compactification for holomorphic maps of degree $d>2 g-2$ into projective space has the structure of a non-singular projective variety.

The proof of Theorem 4.4 is somewhat lengthy, so we will split it into several lemmas. Our first goal is to define a map

$$
u: \mathcal{B}(d, 1, k) \longrightarrow \overline{\mathcal{M}}_{U}(d, 1, k) .
$$

This is achieved by the following

Lemma 4.6. Given a stable $k$-pair $(L, \vec{\phi}) \in \mathcal{B}(d, 1, k)$ there is a unique effective divisor $D \in C_{l}$ and holomorphic map $f_{(L, \vec{\phi})}: C \backslash D \longrightarrow \mathbb{P}^{k-1}$ which extends uniquely to a holomorphic map of degree $d-l$ on $C$.

Proof. Given $(L, \vec{\phi}) \in \mathcal{B}(d, 1, k)$, recall the map $C \rightarrow \mathbb{P}^{k-1}$ given by (2.6). This defines an algebraic map on $C \backslash D$, where $D=\{p \in C: \vec{\phi}(p)=0\}$, counted with multiplicity. By the properness of $\mathbb{P}^{k-1}$ this map extends to a holomorphic map

$$
f_{(L, \vec{\phi})}: C \longrightarrow \mathbb{P}^{k-1},
$$

of degree $d-l$, where $l=\operatorname{deg} D$.

Definition 4.8. The map $u$ of $(4.5)$ is defined by setting $u[L, \vec{\phi}]=\left(D, f_{(L, \vec{\phi})}\right)$, where $D$ and $f_{(L, \vec{\phi})}$ are defined by Lemma 4.6 .

In order to prove Theorem 4.4 it is convenient first to write a local expression for the map (4.7) in terms of the homogeneous coordinates of $\mathbb{P}^{k-1}$. Let $z$ be a local coordinate centered at $p \in D$, and suppose that $m$ is the minimal order of vanishing of the $\phi_{i}$ 's at $p$. Then in a deleted neighborhood of $p$, the map (2.6) is clearly equivalent to

$$
f_{(L, \vec{\phi})}(z)=\left[\frac{\phi_{1}(z)}{z^{m}}, \ldots, \frac{\phi_{k}(z)}{z^{m}}\right]
$$

Since for some $i, \lim _{z \rightarrow 0} \phi_{i}(z) / z^{m} \neq 0,(4.9)$ is the desired extension.

After this small digression, we continue with

Lemma 4.10. The map $u$ is a bijection.

Proof. A set theoretic inverse to $u$ can be constructed as follows: Let $S^{*} \rightarrow \mathbb{P}^{k-1}$ denote the anti-tautological bundle with $k$ tautological sections $z_{1}, \ldots, z_{k}$ given by the coordinates of $\mathbb{C}^{k}$. Given $(D, f) \in \overline{\mathcal{M}}_{U}(d, 1, k)$, set $L=f^{*} S^{*} \otimes \mathcal{O}_{C}(D)$, where $\mathcal{O}_{C}$ denotes the structure sheaf of $C$, and $\phi_{i}=f^{*} z_{i} \otimes 1_{D}, i=1, \ldots, k$, where $1_{D}$ denotes a choice of holomorphic section of $\mathcal{O}_{C}(D)$ vanishing at precisely $D$. Then it is clear that $u^{-1}(D, f)=\left[L, \phi_{1}, \ldots, \phi_{k}\right]$. Observe that a different choice of $1_{D}$ amounts to a rescaling of the $\phi_{i}$ 's and so does not affect the definition of $u^{-1}$. 
The next step is to prove

Lemma 4.11. The map $u$ is continuous.

Proof. Assume $\left[\bar{\partial}_{n}, \vec{\phi}_{n}\right] \rightarrow[\bar{\partial}, \vec{\phi}]$ in $\mathcal{B}(d, 1, k)$. Write $\left(D_{n}, f_{n}\right)=u\left[\bar{\partial}_{n}, \vec{\phi}_{n}\right]$ and $(D, f)=u[\bar{\partial}, \vec{\phi}]$. Since $\pi: \mathcal{B}(d, 1, k) \stackrel{\pi}{\longrightarrow} J_{d}$ is continuous, we may assume that the operators $\bar{\partial}_{n} \rightarrow \bar{\partial}$ on $L$ and that we can choose local holomorphic trivializations simultaneously for all $\bar{\partial}_{n}, \bar{\partial}$. According to the definition of the Uhlenbeck topology we must show

(i) $f_{n} \rightarrow f$ in $C_{0}^{\infty}(C \backslash D)$, and

(ii) $e\left(f_{n}\right)+\delta_{D_{n}} \rightarrow e(f)+\delta_{D}$ as distributions

For (i), pick $p \in C \backslash D$. Clearly, $p \in C \backslash D_{n}$ for $n$ sufficiently large. Then by the construction in Lemma 4.6,

$$
f_{n}(p)=\left[\phi_{1, n}(p), \ldots, \phi_{k, n}(p)\right], \quad f(p)=\left[\phi_{1}(p), \ldots, \phi_{k}(p)\right],
$$

where $\phi_{i, n} \rightarrow \phi_{i}$ smoothly as $n \rightarrow \infty$ for all $i=1, \ldots, k$. This clearly implies (i).

To prove part (ii), recall that the Fubini-Study metric on $\mathbb{P}^{k-1}$ is given by

$$
\omega=\frac{\sqrt{-1}}{2 \pi} \partial \bar{\partial} \log \left(\sum_{i=1}^{k}\left|z_{i}\right|^{2}\right) .
$$

Since $f_{n}$ and $f$ are holomorphic,

$$
\begin{gathered}
e\left(f_{n}\right)(p)=f_{n}^{*} \omega=\frac{\sqrt{-1}}{2 \pi} \partial \bar{\partial} \log \left(\sum_{i=1}^{k}\left|\phi_{i, n}(p)\right|^{2}\right) \\
e(f)(p)=f^{*} \omega=\frac{\sqrt{-1}}{2 \pi} \partial \bar{\partial} \log \left(\sum_{i=1}^{k}\left|\phi_{i}(p)\right|^{2}\right) .
\end{gathered}
$$

Let $z$ be a local coordinate about a point $p \in D$, and let $V$ be a neighborhood of $p$ satisfying $V \cap D=\{p\}$. Furthermore, by induction on $d$ we assume $p \notin D_{n}$ for $n$ large and that the multiplicity $m$ of $p$ in $D$ is $m \geq 1$. Let $g \in C_{0}^{\infty}(V)$. Then

$$
\begin{aligned}
\int_{V} g e\left(f_{n}\right) & =\int g \frac{\sqrt{-1}}{2 \pi} \partial \bar{\partial} \log \left(\sum_{i=1}^{k}\left|\phi_{i, n}(z)\right|^{2}\right) \\
& =\int \partial \bar{\partial} g \frac{\sqrt{-1}}{2 \pi} \log |z|^{2 m}+\int \partial \bar{\partial} g \frac{\sqrt{-1}}{2 \pi} \log \left(\sum_{i=1}^{k}\left|\frac{\phi_{i, n}(z)}{z^{m}}\right|^{2}\right) \\
& =m \delta_{p}(g)+\frac{\sqrt{-1}}{2 \pi} \int_{V} \partial \bar{\partial} g \log \left(\sum_{i=1}^{k}\left|\frac{\phi_{i, n}(z)}{z^{m}}\right|^{2}\right)
\end{aligned}
$$

On the other hand, since $\phi_{i, n}(z) \rightarrow z^{m} \phi_{i}(z)$ in $C_{0}^{\infty}(V)$ for all $i=1, \ldots, k$, it follows from the dominated convergence theorem and the fact that $\log |z|^{-m} \in L^{1}(V)$ that

$$
\log \left(\sum_{i=1}^{k}\left|\frac{\phi_{i, n}(z)}{z^{m}}\right|^{2}\right) \longrightarrow \log \left(\sum_{i=1}^{k}\left|\phi_{i}(z)\right|^{2}\right)
$$


in $L^{1}(V)$. By taking limits in (4.12) we obtain

$$
\int_{V} g e\left(f_{n}\right) \longrightarrow m \delta_{p}(g)+\int_{V} g e(f) .
$$

Now by covering $C$ with $V$ 's as above and using partitions of unity we obtain the convergence (ii). This completes the proof of Lemma 4.6.

Proof of Theorem 4.4. According to Lemmas 4.10 and 4.11, the map $u: \mathcal{B}(d, 1, k) \rightarrow$ $\overline{\mathcal{M}}_{U}(d, 1, k)$ is a continuous bijection of compact topological spaces, and hence is a homeomorphism. It follows that $\overline{\mathcal{M}}_{U}(d, 1, k)$ inherits the projective bundle structure of $\mathcal{B}(d, 1, k)$.

Next, we proceed to show that $\overline{\mathcal{M}}_{U}(d, r, k)$ has the structure of a projective variety for any $r$. Actually, since we are interested in computing intersections, we will have to be more precise and define a scheme structure on $\overline{\mathcal{M}}_{U}(d, r, k)$. The reasons for this will become apparent in the following subsection.

To begin, note that the Plücker embedding

$$
G(r, k) \hookrightarrow \mathbb{P}^{N-1},
$$

where $N=\left(\begin{array}{l}k \\ r\end{array}\right)$, induces an inclusion on the Uhlenbeck spaces

$$
\overline{\mathcal{M}}_{U}(d, r, k) \hookrightarrow \overline{\mathcal{M}}_{U}(d, 1, N)
$$

The next proposition is immediate from the definition of the Uhlenbeck topology:

Proposition 4.15. The inclusion (4.14) is a homeomorphism of $\overline{\mathcal{M}}_{U}(d, r, k)$ onto a closed subspace of $\overline{\mathcal{M}}_{U}(d, 1, N)$.

We now show that the image of (4.14) has a natural scheme structure. We shall use the identification $\overline{\mathcal{M}}_{U}(d, 1, N) \simeq \mathcal{B}(d, 1, N)$ coming from Theorem 4.4. Let $\mathcal{H}=\mathcal{H}(d, 1, N)$ denote the space of holomorphic $k$-pairs $\left(L, \phi_{1}, \ldots, \phi_{N}\right)$, where $L$ is a holomorphic line bundle of degree $d$. On $C \times \mathcal{H}$ we have the universal line bundle $\widetilde{U}$. Suppose $\Xi$ is quadratic form on $\mathbb{C}^{N}$, and denote by $Q_{\Xi}$ the quadric hypersurface in $\mathbb{P}^{N-1}$ defined by $\Xi$. Then $\Xi$ determines a section $\tilde{\psi}_{\Xi}$ of $\widetilde{U}^{\otimes 2}$ as follows: For a point $x=(p ; L, \vec{\phi}) \in C \times \mathcal{H}$, let $\tilde{\psi}_{\Xi}(x)=\Xi(\vec{\phi}(p), \vec{\phi}(p))$. Clearly, $\tilde{\psi}_{\Xi}$ is holomorphic, and since $\Xi$ is quadratic, $\tilde{\psi}_{\Xi}$ is a section of $U^{\otimes 2}$. Moreover, it follows by definition of the action of $\mathfrak{G}^{\mathbb{C}}$ on $\widetilde{U}$ that $\tilde{\psi}_{\Xi}$ is equivariant with respect to this action. Therefore, $\tilde{\psi}_{\Xi}$ descends to a holomorphic section of $U^{\otimes 2} \rightarrow C \times \mathcal{B}(d, 1, N)$. We denote this section by $\psi_{\Xi}$.

Given a point $p \in C$, let $Z_{\Xi}(p)$ denote the zero scheme of $\left.\psi_{\Xi}\right|_{\{p\} \times \mathcal{B}(d, 1, N)}$ in $\mathcal{B}(d, 1, N)$. Then we have the following

Lemma 4.16. Let $p_{1}, \ldots, p_{m}$ be distinct points in $C$ with $m \geq 2 d+1$. Let $Z_{\Xi}$ be the scheme theoretic intersection $Z_{\Xi}\left(p_{1}\right) \cap \cdots \cap Z_{\Xi}\left(p_{m}\right)$. Then $Z_{\Xi}$ corresponds set theoretically to the set of $(D, f) \in \overline{\mathcal{M}}_{U}(d, 1, N)$ where $f(C) \subset Q_{\Xi}$.

Proof. If $f(C) \subset Q_{\Xi}$, then the point $x=[L, \vec{\phi}]$ corresponding to $f$ satisfies $\vec{\phi}(p)=0$ if $p \in D$ and $\left[\phi_{1}(p), \ldots, \phi_{N}(p)\right] \in$ ker $\Xi$ otherwise; in particular, $x \in Z_{\Xi}$. Conversely, suppose $x \in Z_{\Xi}$, and let $(D, f)$ be the point in $\overline{\mathcal{M}}_{U}(d, 1, N)$ corresponding to $x$. Now $Z_{\Xi}\left(p_{i}\right)$ consists of all points $[L, \vec{\phi}]$ where either $\vec{\phi}\left(p_{i}\right)=0$ or 
$\left[\phi_{1}\left(p_{i}\right), \ldots, \phi_{N}\left(p_{i}\right)\right] \in$ ker $\Xi$. If $x \in Z_{\Xi}\left(p_{i}\right)$ then the former condition implies $p_{i} \in D$ and the latter implies $f\left(p_{i}\right) \in Q_{\Xi}$. Either way, $f$ maps at least $m-l$ points into $Q_{\Xi}$, where $l$ is the degree of $D$. Since $f$ has degree $d-l$ and $m-l>2(d-l)$, Bezout's Theorem implies $f(C) \subset Q_{\Xi}$. This completes the proof.

The embedding (4.13) realizes $G(r, k)$ as the common zero locus of quadratic forms $\Xi_{i}, i=1, \ldots, N$ (see $[\mathrm{G}-\mathrm{H}]$, p. 211). Therefore, Lemma 4.16 immediately implies

Theorem 4.17. The image of $\overline{\mathcal{M}}_{U}(d, r, k)$ in $\mathcal{B}(d, 1, N)$ is precisely the intersection $Z_{\Xi_{1}} \cap \cdots \cap Z_{\Xi_{N}}$. In particular, $\overline{\mathcal{M}}_{U}(d, r, k)$ has the structure of a projective scheme.

$\S 4.2$ The Grothendieck Quot scheme. In this section, we will exhibit a different compactification of the space $\mathcal{M}$ in terms of a certain Grothendieck Quot scheme. This compactification is perhaps more natural in the algebraic category and will be essential for our computations in $\S 5$.

Let $\mathcal{F}$ be a coherent sheaf on our fixed Riemann surface $C$. As before, we denote the structure sheaf of $C$ by $\mathcal{O}_{C}$. For each $t \in \mathbb{Z}$, the coherent sheaf Euler characteristic

$$
h_{\mathcal{F}}(t):=\chi(C, \mathcal{F}(t p))=h^{0}(C, \mathcal{F}(t p))-h^{1}(C, \mathcal{F}(t p))
$$

does not depend upon the choice of a point $p \in C$, and $h_{\mathcal{F}}(t)$ is a polynomial in $t$ (see [Gro]). This is referred to as the Hilbert polynomial of the sheaf $\mathcal{F}$. For example, if $E$ is a vector bundle of degree $d$ and rank $r$ on $C$, then by the RiemannRoch theorem, $h_{E}(t)=d+r t-r(g-1)$. In particular, both the rank and degree of a vector bundle are determined by its Hilbert polynomial.

Recall that on the Grassmannian $G(r, k)$, there is the tautological exact sequence

$$
0 \longrightarrow S \longrightarrow \mathcal{O}_{G}^{k} \longrightarrow Q \longrightarrow 0,
$$

where $\mathcal{O}_{G}^{k}$ is the trivial bundle of rank $k$ on $G(r, k)$ and $S$ and $Q$ are the universal bundles of rank $r$ and $k-r$, respectively. If $f: C \rightarrow G(r, k)$ is a holomorphic map of degree $d$, then the pullback of the tautological quotient yields a quotient $\mathcal{O}_{C}^{k} \rightarrow f^{*} Q \rightarrow 0$ of vector bundles on $C$. Furthermore, since $f^{*} S$ is of rank $r$ and degree $-d$, the Hilbert polynomial

$$
h_{f^{*} Q}(t)=h_{d}(t):=k h_{\mathcal{O}_{C}}(t)-[r t-(d+r(g-1))] .
$$

Actually, to be more precise, the map $f$ determines an equivalence class of quotients $\mathcal{O}_{C}^{k} \rightarrow F \rightarrow 0$, where two such quotients are equivalent if there is an isomorphism of the $F$ 's which carries one quotient to the other. Since such a quotient also clearly determines a map from $C$ to $G(r, k)$, we have the following

Lemma 4.19. The set of degree $d$ holomorphic maps $f: C \rightarrow G(r, k)$ may be identified with the set of equivalence classes of quotients $\mathcal{O}_{C}^{k} \rightarrow F \rightarrow 0$, where $h_{F}(t)=h_{d}(t)$ is as defined above.

The idea behind the Quot scheme compactification is to expand the set of quotients to include quotients $\mathcal{O}_{C}^{k} \rightarrow \mathcal{F} \rightarrow 0$, where $\mathcal{F}$ is a coherent sheaf with Hilbert polynomial $h_{\mathcal{F}}(t)=h_{d}(t)$. Following Grothendieck (see [Gro]), one considers the contravariant "quotient" functor assigning to each scheme $X$ the set of quotients $\mathcal{O}_{C \times X}^{k} \rightarrow \widetilde{\mathcal{F}} \rightarrow 0$ (modulo equivalence) of coherent sheaves on $C \times X$ such that $\widetilde{\mathcal{F}}$ is flat over $X$ with relative Hilbert polynomial $h_{d}(t)$. Grothendieck's theorem is the following: 
Theorem 4.20. The quotient functor is representable by a projective scheme. That is, there is a projective scheme $\overline{\mathcal{M}}_{Q}=\overline{\mathcal{M}}_{Q}(d, r, k)$ together with a universal quotient $\mathcal{O}_{C \times \overline{\mathcal{M}}_{Q}}^{k} \rightarrow \widetilde{\mathcal{F}} \rightarrow 0$ flat over $\overline{\mathcal{M}}_{Q}$ with relative Hilbert polynomial $h_{d}(t)$, such that each of the flat quotients over $X$ defined above is equivalent to the pullback of the universal quotient under a unique morphism from $X$ to $\overline{\mathcal{M}}_{Q}$.

The projective scheme $\overline{\mathcal{M}}_{Q}$ defined above is clearly uniquely determined (up to isomorphism) and is called the Grothendieck Quot scheme. Of course, the closed points of $\overline{\mathcal{M}}_{Q}$ correspond to equivalence classes of quotients on $C$, so the scheme $\overline{\mathcal{M}}_{Q}$ parametrizes such equivalence classes and by Lemma 4.19 , the subset of $\overline{\mathcal{M}}_{Q}$ corresponding to vector bundle quotients parametrizes holomorphic maps $f: C \rightarrow$ $G(r, k)$.

But every point of $\overline{\mathcal{M}}_{Q}$ determines a unique holomorphic map to the Grassmannian via the following

Lemma 4.21. (i) The kernel of a quotient $\mathcal{O}_{C}^{k} \rightarrow \mathcal{F} \rightarrow 0$ is always a vector bundle on $C$. free.

(ii) If $\mathcal{O}_{C \times X}^{k} \rightarrow \widetilde{\mathcal{F}} \rightarrow 0$ is a flat quotient over $C \times X$, then the kernel is locally

Proof. The restriction of the kernel to each $C \times\{x\}$ is a vector bundle, because $C$ is a smooth curve. Flatness then implies that the kernel itself is locally free. (See [N], Lemma 5.4.)

Part (i) of Lemma 4.21 tells us that a quotient $\mathcal{O}_{C}^{k} \rightarrow \mathcal{F} \rightarrow 0$ induces an injection of sheaves $0 \rightarrow E^{*} \rightarrow \mathcal{O}_{C}^{k}$, where $E$ is a vector bundle on $C$ of rank $r$ and degree $d$. Moreover, an equivalence class of quotients clearly corresponds to the analogous equivalence class of injections. But an injection of sheaves induces an injection of the fibers at all but a finite number of points, hence a rational map of $C$ to the Grassmannian. As in Lemma 4.6, this in turn defines a holomorphic map of lower degree from $C$ to $G(r, k)$.

We may therefore interpret the Quot scheme as a fine moduli space for the "injection" functor assigning to $X$ the set of sheaf injections $0 \rightarrow \widetilde{E}^{*} \rightarrow \mathcal{O}_{C \times X}^{k}$, and dualizing, we get the following corollary to Theorem 4.20:

Corollary 4.22. The Quot scheme $\overline{\mathcal{M}}_{Q}$ is a fine moduli space for the functor which assigns to each scheme $X$ the set of sheaf maps $\mathcal{O}_{C \times X}^{k} \rightarrow \widetilde{E}$ (modulo equivalence) subject to the following conditions: $\widetilde{E}$ is locally free, for each closed point $x \in X$ the restriction of $\widetilde{E}$ to $C \times\{x\}$ has rank $r$ and degree $d$, and the restriction of the sheaf map to $C \times\{x\}$ is surjective at all but a finite number of points.

If we compare Corollary 4.22 and Definition 3.3, we see that we may interpret the Quot scheme as a fine moduli space for $\tau$-stable $k$-pairs if $\tau>d$ (cf. Proposition 3.14). If $r \geq 2$, these values of $\tau$ are outside the admissible range, so the Quot scheme does not coincide with one of the smooth moduli spaces constructed in $\S 3$. Indeed, the Quot scheme has singularities in general. However, in the rank one case, we have the following:

Corollary 4.23. The Quot scheme $\overline{\mathcal{M}}_{Q}(d, 1, k)$ is isomorphic to the projective bundle $\overline{\mathcal{M}}_{P}(d, k)$ over $J_{d}$ defined in (2.4). 
Proof. By Corollary 4.22 and Theorem 3.5, the moduli space of stable $k$-pairs for rank one bundles is a fine moduli space representing the same functor as $\overline{\mathcal{M}}_{Q}(d, 1, k)$. Therefore they are isomorphic, and by Theorem $3.22, \mathcal{B}(d, 1, k)$ is isomorphic to $\overline{\mathcal{M}}_{P}(d, k)$.

Putting Corollary 4.23 together with Theorem 4.16, we get the following:

Theorem 4.24. There is an algebraic surjection $u: \overline{\mathcal{M}}_{Q}(d, r, k) \rightarrow \overline{\mathcal{M}}_{U}(d, r, k)$ which is an isomorphism on $\mathcal{M}(d, r, k)$.

Proof. Let $N=\left(\begin{array}{l}k \\ r\end{array}\right)$ and consider the universal sheaf map from Corollary 4.21: $\vec{\Psi}: \mathcal{O}_{C \times \overline{\mathcal{M}}_{Q}}^{k} \rightarrow \widetilde{E}$. The map $\wedge^{r}(\Psi): \mathcal{O}_{C \times \overline{\mathcal{M}}_{Q}}^{N} \rightarrow \wedge^{r} \widetilde{E}$ determines a morphism

$$
w: \overline{\mathcal{M}}_{Q}(d, r, k) \longrightarrow \overline{\mathcal{M}}_{Q}(d, 1, N)
$$

Using Corollary 4.23 , the image of $w$ is easily seen to be precisely $\overline{\mathcal{M}}_{U}(d, r, k)$. Now the morphism $w$ is not in general an embedding. However, if $x \in \overline{\mathcal{M}}_{Q}(d, r, k)$ parametrizes a surjective map $\mathcal{O}_{C}^{k} \rightarrow E$, then as we saw above the quotient is completely determined by the corresponding map to $G(r, k)$. Via the Plücker embedding of the Grassmannian, it follows that $w$ can be inverted on the image of $\mathcal{M}(d, r, k)$, so $w$ is an embedding when restricted to $\mathcal{M}(d, r, k)$, which completes the proof of the theorem.

If $D$ is an effective divisor on $C$ of degree $\delta$, let $\widetilde{E}(D)$ denote the tensor product $\widetilde{E} \otimes \pi^{*} \mathcal{O}_{C}(D)$ of bundles on $C \times \overline{\mathcal{M}}_{Q}$. If we let $\tilde{d}=d+r \delta$, then the natural sheaf map $\mathcal{O}_{C} \rightarrow \mathcal{O}_{C}(D)$ induces a map on $C \times \overline{\mathcal{M}}_{Q}$ :

$$
\mathcal{O}_{C \times \overline{\mathcal{M}}_{Q}}^{k} \longrightarrow \widetilde{E} \longrightarrow \widetilde{E}(D)
$$

which, in turn, induces an embedding $\overline{\mathcal{M}}_{Q}(d, r, k) \hookrightarrow \overline{\mathcal{M}}_{Q}(\tilde{d}, r, k)$, since $\widetilde{E}(D)$ is a bundle of rank $r$ and degree $\tilde{d}$. What is less obvious is the following.

Theorem 4.26. (i) For each $\delta>>0$, there is a choice of $\tau$ so that there is an embedding

$$
\overline{\mathcal{M}}_{Q}(d, r, k) \hookrightarrow \mathcal{B}_{\tau}(\tilde{d}, r, k),
$$

where $\mathcal{B}_{\tau}(\tilde{d}, r, k)$ is smooth of dimension $\tilde{d} k-r(k-r)(g-1)$ (see Theorem 3.20).

(ii) If $\mathcal{B}=\mathcal{B}_{\tau}(\tilde{d}, r, k)$ is chosen as in (i), and $U_{\tau}=U_{\tau}(\tilde{d}, r, k)$ is the universal rank $r$ bundle on $C \times \mathcal{B}_{\tau}$, then the embedding in (i) may be chosen so that the embedded $\overline{\mathcal{M}}_{Q}(d, r, k)$ is the scheme-theoretic intersection of $k \delta$ subvarieties, each of which is the zero-scheme of a map $\left.\mathcal{O}_{\mathcal{B}} \rightarrow U_{\tau}\right|_{\{p\} \times \mathcal{B}_{\tau}}$.

Proof. In Theorem 3.20, we showed that for generic $\tau$ in the range given by Assumption 3.12 , the moduli space $\mathcal{B}_{\tau}(\tilde{d}, r, k)$ is smooth, of the expected dimension. Since by Proposition $3.30, \mathcal{B}_{\tau}(\tilde{d}, r, k)$ is a fine moduli space for $\tau$-stable $k$-pairs, we only need to show that the family of $k$-pairs defined by the map (4.25) above is $\tau$-stable for some $\tau$ in the desired range. But for each $x \in \overline{\mathcal{M}}_{Q}(d, r, k)$, the map $\mathcal{O}_{C}^{k} \rightarrow E(D)=\left.\widetilde{E}(D)\right|_{C \times\{x\}}$ factors through $\mathcal{O}_{C}^{k}(D)$ and does not factor through any subbundle of $E(D)$, so if $E(D)$ fits into an exact sequence $0 \rightarrow E^{\prime} \rightarrow E(D) \rightarrow$ $E^{\prime \prime} \rightarrow 0$, then $\mu\left(E^{\prime \prime}\right) \geq \delta$ and the desired $\tau$-stability follows if $\delta>>0$. 
Suppose that $D$ is the sum of $\delta>>0$ distinct points $p_{1}, \ldots, p_{\delta}$ on $C$, and let $\mathcal{B}_{\tau}=$ $\mathcal{B}_{\tau}(\tilde{d}, r, k)$ as above. Then for each $p_{i} \in D$ and each summand $e_{j}: \mathcal{O}_{\mathcal{B}_{\tau}} \hookrightarrow \mathcal{O}_{\mathcal{B}_{\tau}}^{k}$, the universal $k$-pair $\mathcal{O}_{C \times \mathcal{B}_{\tau}}^{k} \rightarrow U_{\tau}$ on $C \times \mathcal{B}_{\tau}$ induces a section: $\Phi_{i, j}:\left.\mathcal{O}_{\mathcal{B}_{\tau}} \rightarrow U_{\tau}\right|_{p_{i} \times \mathcal{B}_{\tau}}$. Let $Z_{i, j} \subset \mathcal{B}_{\tau}$ denote the zero-scheme of $\Phi_{i, j}$, and let

$$
Z=\bigcap_{1 \leq i \leq \delta, 1 \leq j \leq k} Z_{i, j}
$$

If we restrict the universal $k$-pair to $C \times Z$, then the pair factors:

$$
\left.\left.\mathcal{O}_{C \times Z}^{k} \longrightarrow U_{\tau}(-D)\right|_{C \times Z} \longrightarrow U_{\tau}\right|_{C \times Z}
$$

and $\tau$-stability implies that for each $z \in Z$, the restriction of (4.27) to

$$
\left.\mathcal{O}_{C \times\{z\}}^{k} \longrightarrow U_{\tau}(-D)\right|_{C \times\{z\}}
$$

cannot span any subbundle. Thus (4.27) determines a morphism $Z \rightarrow \overline{\mathcal{M}}_{Q}(d, r, k)$ which inverts the map from $\overline{\mathcal{M}}_{Q}(d, r, k)$ to $\mathcal{B}_{\tau}(\tilde{d}, r, k)$, and the theorem is proved.

Although the Quot scheme and Uhlenbeck compactification are not in general smooth, the following theorem and its corollary show that there is at least some reasonable structure to these spaces for large degrees relative to $g, r$, and $k$ (for a sharper description in the case of $g=1$, see $[\mathrm{Br}])$.

Theorem 4.28. There is a function $f(g, r, k)$ such that $\overline{\mathcal{M}}_{Q}(d, r, k)$ is irreducible and generically reduced of the expected dimension $k d-r(k-r)(g-1)$ for all $d \geq$ $f(g, r, k)$. Moreover, for such $d, \mathcal{M}(d, r, k)$ is dense in $\overline{\mathcal{M}}_{Q}(d, r, k)$.

Proof. By induction on the rank $r$ for fixed $g$ and $k$. If $r=1$, then by Corollary 4.23 , the Quot scheme $\overline{\mathcal{M}}_{Q}(d, r, k)$ is a smooth projective bundle of dimension $k d-(k-1)(g-1)$ over $J_{d}$ as soon as $d \geq 2 g-1$. So set $f(g, 1, k)=2 g-1$. For rank two or greater we define

$$
\overline{\mathcal{M}}_{Q}^{s}(d, r, k)=\left\{(E, \vec{\phi}): E \text { is semistable and } \vec{\phi}: \mathcal{O}_{C}^{k} \rightarrow E \text { is surjective }\right\} .
$$

Then $\overline{\mathcal{M}}_{Q}^{s}(d, r, k)$ is simultaneously an open subscheme of $\overline{\mathcal{M}}_{Q}(d, r, k)$ and of $\mathcal{B}_{\tau}(d, r, k)$ for any admissible $\tau$, and for large $d$ it is clearly non-empty (cf. [N]). Recall that all the $\mathcal{B}_{\tau}$ 's are smooth and irreducible, of the expected dimension. Since every component of the Quot scheme always has at least the expected dimension (see [Gro]), the theorem will follow once we show that every component of the complement $\overline{\mathcal{M}}_{Q}(d, r, k) \backslash \overline{\mathcal{M}}_{Q}^{s}(d, r, k)$ has dimension smaller than $k d-r(k-r)(g-1)$.

In order to bound the dimensions of this complement we first recall that if $E$ is unstable then it fits into an exact sequence $0 \rightarrow S \rightarrow E \rightarrow Q \rightarrow 0$, where $S$ is stable, of slope greater than $d / r$. Moreover, if $\vec{\phi}: \mathcal{O}_{C}^{k} \rightarrow E$ generically generates $E$ then the induced section $\mathcal{O}_{C}^{k} \rightarrow Q$ must also generically generate. Finally, if $d_{s}$, $r_{s}$ and $d_{q}, r_{q}$ denote the degrees and ranks of $S$ and $Q$, respectively, because this exact sequence exhibits the instability of $E$ we have $d_{s} r_{q}-d_{q} r_{s}>0$. Thus, any $k$-pair $(E, \vec{\phi})$ in the complement $\overline{\mathcal{M}}_{Q} \backslash \overline{\mathcal{M}}_{Q}^{s}$ may be constructed as follows:

(i) Choose non-negative integers $d_{s}, r_{s}$ and $d_{q}, r_{q}$ such that $d_{s}+d_{q}=d$, $r_{s}+r_{q}=r$, and $d_{s} r_{q}-d_{q} r_{s}>0$.

(ii) Choose a stable bundle $S \in \mathcal{N}\left(d_{s}, r_{s}\right)$ and $(Q, \vec{\varphi}) \in \overline{\mathcal{M}}_{Q}\left(d_{q}, r_{q}, k\right)$.

(iii) Choose an extension $x \in \operatorname{Ext}^{1}(Q, S)$ together with a lift of the sections $\vec{\varphi}$ to sections $\vec{\phi}$ of the bundle $E$ in the resulting exact sequence. 
The main point is that the choice in (iii) comes from a projective space. To be precise, if we let $V$ be defined by the long exact sequence:

$$
0 \rightarrow \operatorname{Hom}(Q, S) \rightarrow \operatorname{Hom}\left(\mathcal{O}_{C}^{k}, S\right) \rightarrow V \rightarrow \operatorname{Ext}^{1}(Q, S) \rightarrow \operatorname{Ext}^{1}\left(\mathcal{O}_{C}^{k}, S\right),
$$

then the choice in (iii) is a point in $\mathbb{P}(V)$. Furthermore, since $S$ is stable with slope at least $d / r$, we may assume that $\operatorname{Ext}^{1}\left(\mathcal{O}_{C}, S\right)=0$, so we have

$$
\begin{aligned}
\operatorname{dim} V & =k \chi(S)-\chi\left(Q^{*} \otimes S\right) \\
& =k\left(d_{s}-r_{s}(g-1)\right)-\left(d_{s} r_{q}-d_{q} r_{s}\right)+r_{s} r_{q}(g-1) .
\end{aligned}
$$

There are only finitely many choices in (i), so it suffices to count dimensions for each choice of $d_{s}, r_{s}, d_{q}, r_{q}$. We distinguish two cases.

Case 1. Suppose $d_{q} \geq f\left(g, r_{q}, k\right)$. Then $\overline{\mathcal{M}}_{Q}\left(d_{q}, r_{q}, k\right)$ has dimension $k d_{q}-$ $r_{q}\left(k-r_{q}\right)(g-1)$ by induction, so the dimension of the component coming from the choice of $d_{s}, r_{s}, d_{q}, r_{q}$ is

$$
\begin{aligned}
\operatorname{dim} \overline{\mathcal{M}}_{Q}\left(d_{q}, r_{q}, k\right)+ & \operatorname{dim} \mathcal{N}\left(d_{s}, r_{s}\right)+\operatorname{dim} \mathbb{P}(V) \\
= & k d_{q}-r_{q}\left(k-r_{q}\right)(g-1)+r_{s}^{2}(g-1)+1 \\
& +k\left(d_{s}-r_{s}(g-1)\right)-\left(d_{s} r_{q}-d_{q} r_{s}\right)+r_{s} r_{q}(g-1)-1 \\
= & k d-r(k-r)(g-1)-\left(d_{s} r_{q}-d_{q} r_{s}\right)-r_{s} r_{q}(g-1),
\end{aligned}
$$

which gives the desired bound, since we are assuming that $g \geq 1$.

Case 2. Suppose $d_{q}<f\left(g, r_{q}, k\right)$. Let $f=f\left(g, r_{q}, k\right)$. In this case, we do not know the dimension of $\overline{\mathcal{M}}_{Q}\left(d_{q}, r_{q}, k\right)$. However, by the reasoning preceding Theorem 4.24 , the dimension is bounded above by $k f-r_{q}\left(k-r_{q}\right)(g-1)$. Hence, by the same calculation as in Case 1, we see that

$$
\begin{aligned}
\operatorname{dim} & \overline{\mathcal{M}}_{Q}\left(d_{q}, r_{q}, k\right)+\operatorname{dim} \mathcal{N}\left(d_{s}, r_{s}\right)+\operatorname{dim} \mathbb{P}(V) \\
& \leq k\left(d_{s}+f\right)-r(k-r)(g-1)-\left(d_{s} r_{q}-d_{q} r_{s}\right)-r_{s} r_{q}(g-1) \\
& =k d-r(k-r)(g-1)+k\left(f-r_{q}\right)-\left(d_{s} r_{q}-d_{q} r_{s}\right)-r_{s} r_{q}(g-1) .
\end{aligned}
$$

But $k\left(f-r_{q}\right) \leq k f$ is bounded, and since we assumed that $d_{q}<f$, the term $d_{s} r_{q}-d_{q} r_{s}$ grows with $d$. For sufficiently large $d$ we therefore have the desired inequality. This completes the proof.

The following is a direct consequence of Theorems 4.24 and 4.28:

Corollary 4.29. For $d \geq f(g, r, k)$ the Uhlenbeck compactification $\overline{\mathcal{M}}_{U}(d, r, k)$ is irreducible and generically reduced. Moreover, for such $d, \mathcal{M}(d, r, k)$ is dense in $\overline{\mathcal{M}}_{U}(d, r, k)$.

\section{INTERSECTION NUMBERS}

$\S 5.1$ Definitions. In this section, we rigorously define the intersection pairings (1.2) of the Introduction. We show, in particular, that when the degree $d$ is sufficiently large these pairings correspond to the "definition" in (1.2) when $\mathcal{M}=$ $\mathcal{M}(d, r, k)$ is compactified by the Grothendieck Quot scheme of $\S 4.2$. We will use 
the following notation for intersections. If $c_{1}, \ldots, c_{n}$ are Chern classes of codimension $d_{i}$ on an irreducible projective scheme $X$ such that $\sum_{i=1}^{n} d_{i}=\operatorname{dim} X$, then we will denote by $\left\langle c_{1} \cdots c_{n} ; X\right\rangle$ the intersection pairing of the $c_{i}$ 's with $X$. This is a well-defined integer, even if $X$ is not smooth. We refer to Fulton [Ful] for details.

Recall that the evaluation map $\mu: C \times \mathcal{M} \rightarrow G(r, k)$ of (1.1) defines Chern classes $X_{1}, \ldots, X_{r}$ on $\mathcal{M}$ by pulling back the Chern classes of the tautological bundle $S^{*}$ on $G(r, k)$ and restricting to $\{p\} \times \mathcal{M}$. Since the Quot scheme $\overline{\mathcal{M}}_{Q}=\overline{\mathcal{M}}_{Q}(d, r, k)$ admits a universal rank $r$ bundle $\tilde{E}$ on $C \times \overline{\mathcal{M}}_{Q}$ which extends the pullback of $S^{*}$, we immediately obtain the following

Lemma 5.1. The Chern classes $c_{i}:=c_{i}\left(\left.\tilde{E}\right|_{\{p\} \times \overline{\mathcal{M}}_{Q}}\right)$ on $\overline{\mathcal{M}}_{Q}$ restrict to the classes $X_{1}, \ldots, X_{r}$ on $\mathcal{M}$.

Thus, as a first approximation one might expect the correct definition of the intersection numbers (1.2) to be the following. For any set of integers $s_{1}, \ldots, s_{r}$ such that $\sum_{i=1}^{r} i s_{i}=\operatorname{dim} \overline{\mathcal{M}}_{Q}$ one defines

$$
\left\langle X_{1}^{s_{1}} \cdots X_{r}^{s_{r}}\right\rangle:=\left\langle c_{1}^{s_{1}} \cdots c_{r}^{s_{r}} ; \overline{\mathcal{M}}_{Q}\right\rangle .
$$

Unfortunately, there are problems with this definition. It may be the case that $\mathcal{M}$ has many components of different dimension, or one component of dimension different than the expected dimension $k d-r(k-r)(g-1)$ found in $\S 3$. Or, the space $\mathcal{M}$ may have the expected dimension but the "compactification" given by the Quot scheme (or the Uhlenbeck space) may contribute extra components, perhaps even of the wrong dimension, which should not be counted in the intersection numbers.

On the other hand, we showed in Theorem 4.28 that for sufficiently large values of $d$ the Quot scheme $\overline{\mathcal{M}}_{Q}(d, r, k)$ is irreducible and generically reduced of the expected dimension. Thus, it follows that (5.2) is a reasonable definition of the intersection numbers for large $d$. We can then use this fact to construct a good definition for all $d$ as follows:

Lemma 5.3. If $D \subset C$ is an effective divisor of degree $\delta$ chosen so that $\overline{\mathcal{M}}_{Q}(d+r \delta, r, k)$ is irreducible and generically reduced, let

$$
\overline{\mathcal{M}}_{Q}(d, r, k) \hookrightarrow \overline{\mathcal{M}}_{Q}(d+r \delta, r, k)
$$

be the embedding defined in Theorem 4.26. Then the pairing

$$
\left\langle c_{1}^{s_{1}} \cdots c_{r-1}^{s_{r-1}} c_{r}^{s_{r}+k \delta} ; \overline{\mathcal{M}}_{Q}(d+r \delta, r, k)\right\rangle
$$

is independent of the choice of $D$.

Proof. Suppose $\overline{\mathcal{M}}_{Q}(d)=\overline{\mathcal{M}}_{Q}(d, r, k)$ is irreducible of the correct dimension, and $D=\{q\}$. Then the restriction of the universal bundle $\tilde{E}$ on $C \times \overline{\mathcal{M}}_{Q}(d+r)$ to $C \times \overline{\mathcal{M}}_{Q}(d)$ coincides with the twist $\tilde{E}(q)$. Since the Chern classes $c_{1}, \ldots, c_{r}$ are defined by restricting to $\{p\} \times \overline{\mathcal{M}}_{Q}$, the $c_{i}$ 's extend without change from $\overline{\mathcal{M}}_{Q}(d)$ to $\overline{\mathcal{M}}_{Q}(d+r)$. In addition, using the universal sections $\vec{\phi}: \mathcal{O}^{k} \rightarrow \tilde{E}$, we see as in Theorem 4.26 (i) that the image of $\overline{\mathcal{M}}_{Q}(d)$ in $\overline{\mathcal{M}}_{Q}(d+r)$ may be described as the intersection of the zero loci of the sections $\phi_{j}: \mathcal{O} \rightarrow \tilde{E}_{\{q\} \times \overline{\mathcal{M}}_{Q}(d+r)}$. The zero loci are necessarily regular because of the dimension of $\overline{\mathcal{M}}_{Q}(d)$. This proves the 
lemma in this special case. More generally, suppose $\overline{\mathcal{M}}_{Q}(d), D$, and $D^{\prime}$ are given, with $\overline{\mathcal{M}}_{Q}(d+r \delta)$ and $\overline{\mathcal{M}}_{Q}\left(d+r \delta^{\prime}\right)$ irreducible and generically reduced. Then we may embed $\overline{\mathcal{M}}_{Q}(d)$ in $\overline{\mathcal{M}}_{Q}\left(d+r\left(\delta+\delta^{\prime}\right)\right)$ by passing through either $\overline{\mathcal{M}}_{Q}(d+r \delta)$ or $\overline{\mathcal{M}}_{Q}\left(d+r \delta^{\prime}\right)$. But the special case of the lemma implies that the intersection numbers in both cases coincide with

$$
\left\langle c_{1}^{s_{1}} \cdots c_{r-1}^{s_{r-1}} c_{r}^{s_{r}+k\left(\delta+\delta^{\prime}\right)} ; \overline{\mathcal{M}}_{Q}\left(d+r\left(\delta+\delta^{\prime}\right)\right)\right\rangle .
$$

This completes the proof.

We therefore take (5.4) to be the definition of the intersection numbers. Next we will show that the intersection numbers may be computed on the smooth moduli spaces $\mathcal{B}_{\tau}$ for certain choices of $\tau$. This will be essential for the computations in $\S 5.3$.

Theorem 5.5. Let $\overline{\mathcal{M}}_{Q}(d) \hookrightarrow \mathcal{B}_{\tau}(d+r \delta)$ be an embedding from Theorem 4.26 (i). Let $c_{i}:=c_{i}\left(\left.U_{\tau}\right|_{\{p\} \times \mathcal{B}_{\tau}}\right)$, where $U_{\tau}$ is the universal rank $r$ bundle on $C \times \mathcal{B}_{\tau}$. Then

$$
\left\langle X_{1}^{s_{1}} \cdots X_{r}^{s_{r}}\right\rangle=\left\langle c_{1}^{s_{1}} \cdots c_{r-1}^{s_{r-1}} c_{r}^{s_{r}+k \delta} ; \mathcal{B}_{\tau}(d+r \delta)\right\rangle
$$

where $\delta$ is as in Theorem 4.26.

Proof. If $\overline{\mathcal{M}}_{Q}(d)$ is irreducible of the expected dimension, then as in the proof of Lemma 5.3 the $c_{i}$ 's extend unchanged to $\mathcal{B}_{\tau}$, each $Z_{i, j}$ in the intersection $\overline{\mathcal{M}}_{Q}(d)=$ $\bigcap Z_{i, j}$ of Theorem 4.26 (ii) has codimension exactly $r$, and the intersection defines a subvariety of codimension $r k \delta$ in $\mathcal{B}_{\tau}(d+r \delta)$. Therefore, as in the proof of Lemma 5.3 , the pairing $\left\langle c_{1}^{s_{1}}, \ldots, c_{r}^{s_{r}+k \delta} ; \mathcal{B}_{\tau}(d+r \delta)\right\rangle$ is the same as the pairing $\left\langle c_{1}^{s_{1}}, \ldots, c_{r}^{s_{r}} ; \overline{\mathcal{M}}_{Q}(d)\right\rangle$. The general case now follows: If $\overline{\mathcal{M}}_{Q}(d)$ is fixed, let divisors $D$ and $D^{\prime}$ be chosen of degrees $\delta$ and $\delta^{\prime}$, respectively, so that $D$ satisfies the conditions of Theorem 4.26 (i) and so that $\overline{\mathcal{M}}_{Q}\left(d+r \delta^{\prime}\right)$ is irreducible, of the correct dimension. It then follows from the definition of $\tau$-stability that both $\mathcal{B}_{\tau}(d+r \delta)$ and $\overline{\mathcal{M}}_{Q}\left(d+r \delta^{\prime}\right)$ embed in the same space $\mathcal{B}_{\tau^{\prime}}\left(d+r\left(\delta+\delta^{\prime}\right)\right)$, where $\tau^{\prime}=\tau+\delta^{\prime} / r$. But now as in the proof of Lemma 5.3, the Quot scheme $\overline{\mathcal{M}}_{Q}(d)$ embeds in $\mathcal{B}_{\tau^{\prime}}\left(d+r\left(\delta+\delta^{\prime}\right)\right)$ either through $\mathcal{B}_{\tau}$ or through the Quot scheme, and by the special case of the lemma already proved, we see that both intersection numbers are computed by the same pairing of the $c_{i}$ 's on $\mathcal{B}_{\tau^{\prime}}\left(d+r\left(\delta+\delta^{\prime}\right)\right)$.

$\S 5.2$ The conjecture of Vafa and Intriligator. We now pause to describe a conjecture for the intersection numbers defined above which is due to C. Vafa and was worked out in detail by K. Intriligator. The conjecture arises from considerations of certain superconformal field theories. The physical reasoning which led to this prediction may be found in $[\mathrm{V}],[\mathrm{I}]$, and the references therein; here, however, we shall simply give the mathematical formulation of the statement.

We begin by recalling that the ring structure of $H^{*}(G(r, k), \mathbb{C})$ is given by the free ring on the Chern classes of the universal rank $r$ bundle $S$, modulo the ideal of relations obtained by the vanishing of the Segre classes of $S$ beyond the rank of the universal quotient bundle $Q$. More precisely, let $X_{i}=c_{i}\left(S^{*}\right), i=1, \ldots, r$, be as before. From the exact sequence (4.18) we have $c_{t}\left(S^{*}\right) c_{t}\left(Q^{*}\right)=1$, where $c_{t}$ denotes the Chern polynomial. The fact that $Q^{*}$ has rank $k-r$ implies relations on the $X_{i}$; if $I$ denotes the ideal generated by these relations, then we have 
Proposition 5.7. (cf. [B-T], p. 293) There is a ring isomorphism

$$
H^{*}(G(r, k), \mathbb{C}) \simeq \mathbb{C}\left[X_{1}, \ldots, X_{r}\right] / I
$$

Perhaps less well-known is the fact that $I$ is of the form $\left\langle\partial W / \partial X_{i} ; i=1, \ldots, r\right\rangle$, i.e. all the relations are obtained by setting to zero the gradient of some homogeneous polynomial $W$ in the $X_{i}$ 's. To see this, write $c_{t}\left(Q^{*}\right)=\sum_{i=1}^{k} Y_{i}\left(X_{1}, \ldots, X_{r}\right) t^{i}$. Then $I$ is generated by the equations

$$
Y_{i}=0, i=k-r+1, \ldots, k .
$$

On the other hand, making a formal expansion

$$
-\log c_{t}\left(S^{*}\right)=\sum_{j \geq 0} W_{j}\left(X_{1}, \ldots, X_{r}\right) t^{j}
$$

it is easily seen that $Y_{k+1-i}=-\partial W_{k+1} / \partial X_{i}, i=1, \ldots, r$, so that (5.8) implies the relations are generated by the equations $d W_{k+1}=0$. In terms of the Chern roots of $S^{*}$ defined by $c_{t}\left(S^{*}\right)=\prod_{i=1}^{r}\left(1+q_{i} t\right)$, we find that we may take

$$
W=(-1)^{k+1} W_{k+1}=\sum_{i=1}^{r} \frac{q_{i}^{k+1}}{k+1} .
$$

We thus have

Proposition 5.9. Let $W$ be defined as above. Then the ideal I in Proposition 5.7 is generated by the polynomials $\left\{\partial W / \partial X_{i} ; i=1, \ldots, r\right\}$.

In order to state the conjecture we first establish some notation. Let $\widetilde{W}=$ $W+(-1)^{r} X_{1}$, and set

$$
h\left(X_{1}, \ldots, X_{r}\right)=(-1)^{r(r-1) / 2} \operatorname{det}\left(\frac{\partial^{2} W}{\partial X_{i} \partial X_{j}}\right) .
$$

Conjecture 5.10. ([I], eq. (5.5)) The intersection numbers (5.2) are given by

$$
\left\langle X_{1}^{s_{1}} \cdots X_{r}^{s_{r}}\right\rangle=\sum_{d \widetilde{W}\left(Z_{1}, \ldots, Z_{r}\right)=0} h^{g-1}\left(Z_{1}, \ldots, Z_{r}\right) Z_{1}^{s_{1}} \cdots Z_{r}^{s_{r}} .
$$

We shall always interpret Conjecture 5.10 to apply to the case where the degree $d$ is sufficiently large.

Consider, for example, the case $r=1$, i.e. the case of the maps to projective space. The polynomial $W=X^{k+1} / k+1$, and the critical points of $\widetilde{W}$ are the $k$-th roots of unity. Furthermore, $h^{g-1}(Z)=\left(W^{\prime \prime}\right)^{g-1}=k^{g-1} Z^{(k-1)(g-1)}$. Thus we have

$$
\sum_{\widetilde{W}^{\prime}(Z)=0} h^{g-1}(Z) Z^{k d-(k-1)(g-1)}=\sum_{Z^{k}=1} k^{g-1} Z^{k d}=\sum_{Z^{k}=1} k^{g-1}=k^{g} .
$$

By Theorem 2.9, $k^{g}$ is precisely the top intersection of the class in the space of holomorphic maps of degree $d$ to $\mathbb{P}^{k-1}$ defined by $X$. We have established 
Theorem 5.11. Conjecture 5.10 is true for $r=1$.

For the rest of the paper we shall assume $r=2$. The reason for this is that for maps into $G(2, k)$ the results of $\$ 3.3$ and $\$ 5.1$ give us an effective method for computing the left hand side in Conjecture 5.10. In the next subsection we shall set up this calculation for arbitrary genus, however we shall only carry it through in the case of elliptic curves $g=1$. Therefore, let us set

$$
I(d, k ; n)=\sum_{d \widetilde{W}\left(Z_{1}, Z_{2}\right)=0} Z_{1}^{k d-2 n} Z_{2}^{n}
$$

for $n=0,1, \ldots,[k d / 2]$ and $\widetilde{W}$ the polynomial associated to $G(2, k)$ as above. Our goal is to simplify the expression (5.12).

As discussed in $[\mathrm{I}]$, the sum on the right hand side of (5.12) is most clearly expressed in terms of the Chern roots $q_{1}$ and $q_{2}$. The critical points of $\widetilde{W}$ are given by $q_{i}=\alpha \xi_{i}, \alpha^{k}=-1$, where $\xi_{1}$ and $\xi_{2}$ run over the $k$-th roots of unity such that $\xi_{1} \neq \xi_{2}$. This overcounts by a factor of 2 , since the $X_{i}$ 's are symmetric in the $q_{i}$ 's. Thus

$$
\begin{aligned}
I(d, k ; n) & =\frac{(-1)^{d}}{2} \sum_{\substack{\xi_{i}^{k}=1 \\
\xi_{1} \neq \xi_{2}}}\left(\xi_{1}+\xi_{2}\right)^{k d-2 n}\left(\xi_{1} \xi_{2}\right)^{n} \\
& =\frac{(-1)^{d}}{2} \sum_{\xi_{i}^{k}=1}\left(\xi_{1}+\xi_{2}\right)^{k d-2 n}\left(\xi_{1} \xi_{2}\right)^{n}-\frac{(-1)^{d}}{2} \sum_{\xi^{k}=1}(2 \xi)^{k d-2 n} \xi^{2 n} \\
& =(-1)^{d+1} k 2^{k d-2 n-1}-\frac{(-1)^{d+1}}{2} \sum_{\xi_{i}^{k}=1}\left(\xi_{1}+\xi_{2}\right)^{k d-2 n}\left(\xi_{1} \xi_{2}\right)^{n}
\end{aligned}
$$

If we now set $z=\xi_{2} \xi_{1}^{-1}$ we may eliminate one of the roots and introduce a factor of $k$. The result is

$$
\begin{aligned}
I(d, k ; n) & =(-1)^{d+1} k 2^{k d-2 n-1}-(-1)^{d+1} \frac{k}{2} \sum_{z^{k}=1}(1+z)^{k d-2 n} z^{n} \\
& =(-1)^{d+1} k 2^{k d-2 n-1}-(-1)^{d+1} \frac{k}{2} \sum_{q=0}^{k d-2 n} \sum_{z^{k}=1}\left(\begin{array}{c}
k d-2 n \\
q
\end{array}\right) z^{n+q}, \\
(5.13) I(d, k ; n) & =(-1)^{d+1} k 2^{k d-2 n-1}-(-1)^{d+1} \frac{k^{2}}{2} \sum_{\substack{p \in \mathbb{Z} \\
n / k \leq p \leq d-n / k}}\left(\begin{array}{c}
k d-2 n \\
k p-n
\end{array}\right),
\end{aligned}
$$

since the sum of $z^{n+q}$ over the $k$-th roots of unity vanishes unless $n+q$ is of the form $k p$. Equation (5.13) is the desired expression.

$\S 5.3$ Computations. In this section we will outline a procedure for calculating all intersection pairings of the form $\left\langle c_{1}^{m} c_{2}^{n} ; \mathcal{B}_{\tau}(d, 2, k)\right\rangle$ where $\mathcal{B}_{\tau}$ is one of the smooth moduli spaces of $\tau$-stable $k$-pairs from $\S 3$, and $m+2 n=k d-2(k-2)(g-1)=\operatorname{dim} \mathcal{B}_{\tau}$. From these pairings and Theorem 5.5, one recovers all the Gromov invariants for maps from a Riemann surface to $G(2, k)$. We shall compute these invariants in the case where $C$ is elliptic and show that they agree with Conjecture 5.10. 
For each fixed degree $d$, recall that the admissible values of $\tau$ lie in the range $d / 2<\tau<d-(2 g-2)$ (Assumption 3.12) and that if $\left[\tau, \tau^{\prime}\right] \cap \mathbb{Z}=\emptyset$ then $\mathcal{B}_{\tau}=\mathcal{B}_{\tau^{\prime}}$ (see the discussion following Theorem 3.38). Thus, if we fix some $0<\varepsilon<1 / 2$, and list moduli spaces: $\mathcal{B}_{d / 2+\varepsilon}, \mathcal{B}_{l \pm \varepsilon},[d / 2]<l<d-(2 g-2)$, then each smooth moduli space appears in the list, and with the exception of $\mathcal{B}_{d-(2 g-1)+\varepsilon}$, each moduli space is counted exactly twice; once as $\mathcal{B}_{l-\varepsilon}$ and once as $\mathcal{B}_{(l-1)+\varepsilon}\left(\right.$ or $\left.\mathcal{B}_{d / 2+\varepsilon}\right)$.

The calculation of the intersection pairings $\left\langle c_{1}^{m} c_{2}^{n} ; \mathcal{B}_{\tau}\right\rangle$ naturally breaks into two steps. First, an initial calculation is necessary to compute the pairings $\left\langle c_{1}^{m} c_{2}^{n} ; \mathcal{B}_{d / 2+\varepsilon}\right\rangle$. If the degree is odd, then by Theorem 3.26, the moduli space $\mathcal{B}_{d / 2+\varepsilon}$ is a projective bundle over $\mathcal{N}(2, d)$, and the calculation is analogous to the rank one calculation of Theorem 2.9. In principle, this calculation is therefore always computable by Grothendieck-Riemann-Roch since the full cohomology ring structure of $\mathcal{N}(2, d)$ is now known (see $[\mathrm{K}]$, [T1], and [Z]). If the degree is even, a further argument is necessary because of the existence of semistable bundles which are not stable. This can be dealt with without too much difficulty in the genus one case, as we will see below.

Next, there are the flip calculations which compute the difference between the pairings $\left\langle c_{1}^{m} c_{2}^{n} ; \mathcal{B}_{l-\varepsilon}\right\rangle$ and $\left\langle c_{1}^{m} c_{2}^{n} ; \mathcal{B}_{l+\varepsilon}\right\rangle$. This is represented by a class which lives on a "rank one" locus $\overline{\mathcal{M}}_{P}(d-l, k) \times J_{l}$, and thus may be viewed as a rank one calculation. Since every smooth moduli space is a $\mathcal{B}_{l \pm \varepsilon}$, we see that all the pairings, including those which produce the Gromov invariants, are computed by the initial calculation followed by a sequence of flip calculations. We now give the details of these two contributions.

The Initial Calculations. Because the moduli spaces $\mathcal{B}_{d / 2+\varepsilon}$ behave differently depending upon the parity of $d$, we will consider the even and odd cases separately.

Odd degree. If $V$ is any universal bundle on $C \times \mathcal{N}(2, d)$, then by Theorem 3.26 the moduli space $\mathcal{B}_{d / 2+\varepsilon}$ is isomorphic to the projective bundle $\mathbb{P}\left(\left(\rho_{*} V\right)^{\oplus k}\right)$, and if $\pi: \mathcal{B}_{d / 2+\varepsilon} \rightarrow \mathcal{N}(2, d)$ is the projection, then by Corollary 3.32 the universal bundle on $C \times \mathcal{B}_{d / 2+\varepsilon}$ is isomorphic to $\pi^{*} V \otimes \mathcal{O}(1)$ (all pulled back to $C \times \mathcal{B}_{d / 2+\varepsilon}$ ). The intersection pairings now reduce to a calculation involving Grothendieck-RiemannRoch. In particular, we easily obtain

Calculation 5.14. If $C$ is elliptic and $d$ is odd, then $\left\langle c_{1}^{m} c_{2}^{n} ; \mathcal{B}_{d / 2+\varepsilon}\right\rangle=k 2^{m-1}$.

Proof. The moduli space $\mathcal{N}(2, d)$ is isomorphic to $C$ itself in this case. Moreover, if we let $\Delta \subset C \times C$ be the diagonal, then there is a canonical extension on $C \times \mathcal{N}(2,1)$ :

$$
0 \longrightarrow \mathcal{O}_{C \times C} \longrightarrow V \longrightarrow \mathcal{O}_{C \times C}(\Delta) \longrightarrow 0
$$

which produces a universal bundle. If we let $D \subset C$ be a divisor of degree $(d-1) / 2$, then $V(D):=V \otimes \rho^{*} O_{C}(D)$ is a universal bundle on $C \times \mathcal{N}(2, d)$, which satisfies:

$$
\begin{aligned}
\text { (i) } c_{1}\left(V(D)_{p}\right) & =[q], \\
\text { (ii) } c_{1}\left(\rho_{*} V(D)\right) & =((d-1) / 2)[q],
\end{aligned}
$$

where $[q]$ is the class of a point in $\mathcal{N}(2, d)$.

We need to consider the bundle $\left(\pi^{*} V(D) \otimes \mathcal{O}(1)\right)_{p}$. If we let $z$ represent the first Chern class of $\mathcal{O}(1)$, then from (i) and the formula for the Chern classes of a tensor 
product, $c_{1}=\pi^{*}[q]+2 z$ and $c_{2}=\pi^{*}[q] z+z^{2}=c_{1}^{2} / 4$. Thus $c_{1}^{m} c_{2}^{n}=2^{-2 n} c_{1}^{k d}$. If we let $s_{0}$ and $s_{1}$ be the first two Segre classes of $\left(\rho_{*} V(D)\right)^{\oplus k}$, then the intersections are calculated as follows (cf. (2.12)):

$$
\begin{aligned}
c_{1}^{k d} & =\left(\pi^{*}[q]+2 z\right)^{k d}=(k d) 2^{k d-1}\left(s_{0}\right) \pi^{*}[q]+2^{k d}\left(s_{1}\right) \\
& =(k d) 2^{k d-1}-2^{k d} k\left(\frac{d-1}{2}\right)=k 2^{k d-1}
\end{aligned}
$$

and the lemma is proved.

Even degree. Here we unfortunately do not have a simple description of the moduli spaces $\mathcal{B}_{d / 2+\varepsilon}$ in general. In case the genus is one, however, the following proposition will enable us to compute the intersection numbers on a projective variety mapping with degree two onto the moduli space.

Suppose $C$ is elliptic, and let $P=P(d / 2, k)=\overline{\mathcal{M}}_{P}(d / 2, k)$ be the rank one moduli space defined in (2.4). Let $\Delta_{P} \subset P \times P$ be the diagonal, and let $P(2)$ be the blow-up of $P \times P$ along the diagonal. Let $A \subset P(2)$ be the exceptional divisor, and let $\mathcal{L}(2)$ be the pull-back to $C \times P(2)$ of the direct sum of the two obvious universal line bundles on $C \times P(2)$. Finally, let $\mathcal{L}_{A}$ be the pull-back to $C \times A$ of the universal bundle on $C \times \Delta_{P} \cong C \times P$. Then:

Lemma 5.15. (i) There is a holomorphic map $\sigma: P(2) \rightarrow \mathcal{B}_{d / 2+\varepsilon}$, whose fibres are generically finite of degree two.

(ii) There is an exact sequence of sheaves on $C \times P(2)$ :

$$
0 \longrightarrow \mathcal{L}(2) \otimes \mathcal{O}(-C \times A) \longrightarrow \sigma^{*} U_{d / 2+\varepsilon} \longrightarrow \mathcal{L}_{A} \longrightarrow 0
$$

Proof. The diagonal inclusion of line bundles $\mathcal{L} \hookrightarrow \mathcal{L} \oplus \mathcal{L}$ over $C \times \Delta_{P}$ induces the exact sequence of bundles on $C \times A:\left.0 \rightarrow \mathcal{L}_{A} \rightarrow \mathcal{L}(2)\right|_{A} \rightarrow \mathcal{L}_{A} \rightarrow 0$. Denote by $\mathcal{K}$ the kernel of the sheaf map $\mathcal{L}(2) \rightarrow \mathcal{L}_{A}$, where $\mathcal{L}_{A}$ is extended by zero to all of $C \times P(2)$. Then the $k$ sections $\mathcal{O}^{k} \rightarrow \mathcal{L}(2)$ lift to sections of $\mathcal{K}$, so we get in this way a family of pairs parameterized by $P(2)$. One checks that these pairs are all $d / 2+\epsilon$ stable, which gives the map $\sigma$ of (i). A general point of $\mathcal{B}_{d / 2+\epsilon}$ is a pair $(E, \vec{\phi})$, where $E$ is a non-trivial extension $0 \rightarrow L_{1} \rightarrow E \rightarrow L_{2} \rightarrow 0$ of non-isomorphic line bundles $L_{1}$ and $L_{2}$. The preimage of $(E, \vec{\phi})$ under $\sigma$ lives in $P(2)-A$, hence can be thought of as a subset of $P \times P$. But the preimage is easily seen to consist of two reduced points, depending upon whether $L_{1}$ is in the first or second factor.

Since $\mathcal{B}_{d / 2+\epsilon}$ is a fine moduli space, it follows that the pull-back of the universal pair on $\mathcal{B}_{d / 2+\epsilon}$ coincides precisely (i.e. without twisting by a line bundle) with $\mathcal{K}$, and (ii) is obtained.

The following proposition will be essential to both this calculation and the flip calculations which follow.

Proposition 5.16. Suppose $X \subset Y$ is a proper inclusion of smooth, projective varieties of dimensions $M$ and $N$, respectively. Suppose that $E$ is a vector bundle of rank two on $Y$, such that when $E$ is restricted to $X$, there is a surjective map $\left.E\right|_{X} \rightarrow L$ to an invertible sheaf $L$ on $X$. Let $\widetilde{Y}$ denote the blow-up of $Y$ along $X$, let $A \subset \widetilde{Y}$ denote the exceptional divisor, and let $\widetilde{E}$ and $\widetilde{L}$ denote the pullbacks of $E$ and $L$ to $\widetilde{Y}$ and $A$, respectively. Let $i: A \hookrightarrow \widetilde{Y}$ be the inclusion map, and 
finally, suppose $F$ is a rank two bundle fitting in the following exact sequence: $0 \rightarrow \widetilde{E}(-A) \rightarrow F \rightarrow i_{*} \widetilde{L} \rightarrow 0$. Then:

(i) $c_{1}(F)=c_{1}(\widetilde{E})-A$ and $c_{2}(F)=c_{2}(\widetilde{E})-i_{*}\left(c_{1}(\widetilde{L})\right)$.

(ii) If $m, n$ are integers such that $m+2 n=N$, then the integer

$$
\left(c_{1}^{m}(F) c_{2}^{n}(F)-c_{1}^{m}(\widetilde{E}) c_{2}^{n}(\widetilde{E}) ; \widetilde{Y}\right)
$$

is equal to the evaluation on $X$ of the coefficient of $t^{M-n}$ in the polynomial:

$$
-s_{t}(\nu(X))\left(1+c_{1}(E) t\right)^{m}\left(c_{1}(L)+c_{2}(E) t\right)^{n},
$$

where $s_{t}(\nu(X))$ is the Segre polynomial of the normal bundle to $X$ in $Y$.

Proof. (i) is an immediate consequence of the Grothendieck-Riemann-Roch theorem applied to the sequence defining $F$ (see [Ful]). To prove (ii), note first that because of (i), we have

$$
c_{1}^{m}(F) c_{2}^{n}(F)=\left(\sum_{a=0}^{m}\left(\begin{array}{c}
m \\
a
\end{array}\right)(-A)^{a} c_{1}^{m-a}(\widetilde{E})\right)\left(\sum_{b=0}^{n}\left(\begin{array}{c}
n \\
b
\end{array}\right)(-1)^{b}\left(i_{*} c_{1}(\widetilde{L})\right)^{b} c_{2}^{n-b}(\widetilde{E})\right) .
$$

Since with the exception of the $a=b=0$ term, all the terms on the right hand side may be evaluated on $A$, the right hand side becomes:

$$
c_{1}^{m}(\widetilde{E}) c_{2}^{n}(\widetilde{E})+\sum_{b=0}^{n} \sum_{a+b=\max (b, 1)}^{m+b}\left(\begin{array}{c}
m \\
a
\end{array}\right)\left(\begin{array}{c}
n \\
b
\end{array}\right)(-1)(-A)^{a+b-1} c_{1}^{m-a}(\widetilde{E}) c_{2}^{n-b}(\widetilde{E}) c_{1}^{b}(\widetilde{L})
$$

where $c_{1}^{m}(\widetilde{E}) c_{2}^{n}(\widetilde{E})$ is evaluated on $\widetilde{Y}$ and the double sum is evaluated on $A$. Since $m+2 n=N$, we see that the restriction of $(-A)^{a+b-1}$ to $A$ is realized by the Segre class $s_{a+b+M-N}(\nu(X))$ whenever $a+b \geq N-M$. Since the restriction to $A$ of the other classes $c_{1}(\widetilde{E}), c_{2}(\widetilde{E})$ and $c_{1}(\widetilde{L})$ are all pullbacks from $X$, it follows that the terms involving lower powers of $-A$ all evaluate to zero on $A$. Thus we can substitute the appropriate Segre classes for powers of $-A$ and part (ii) immediately follows.

Calculation 5.17. If $C$ is elliptic and $d$ is even, then:

$$
\left\langle c_{1}^{m} c_{2}^{n} ; \mathcal{B}_{d / 2+\varepsilon}\right\rangle=\frac{k^{2}}{2}\left(\begin{array}{c}
k d-2 n \\
k d / 2-n
\end{array}\right)-k 2^{m-1}
$$

Proof. Let $\sigma$ be the degree two map of Lemma 5.15, and, abusing notation slightly, let $c_{1}=\sigma^{*}\left(c_{1}\right)$ and $c_{2}=\sigma^{*}\left(c_{2}\right)$. Then part (i) of Lemma 5.15 implies that the evaluation of $c_{1}^{m} c_{2}^{n}$ on $P(2)$ gives twice the evaluation on $\mathcal{B}_{d / 2+\varepsilon}$.

If we apply Proposition 5.16 to the exact sequence in part (ii) of Lemma 5.15, then we may express $\left\langle c_{1}^{m} c_{2}^{n} ; P(2)\right\rangle$ as the sum of two contributions, the first being

$$
\left\langle c_{1}^{m}\left(\mathcal{L}(2)_{p}\right) c_{2}^{n}\left(\mathcal{L}(2)_{p}\right) ; P(2)\right\rangle
$$

and the second being the evaluation on $\Delta_{P}$ of the coefficient of $t^{m / 2}$ in the polynomial

$$
-s_{t}\left(\nu\left(\Delta_{P}\right)\right)\left(1+c_{1}\left(\mathcal{L}(2)_{p}\right) t\right)^{m}\left(c_{1}(\mathcal{L})+c_{2}(\mathcal{L}(2)) t\right)^{n} .
$$


The first contribution is easy to compute. If we let $\mathcal{L}_{1}$ and $\mathcal{L}_{2}$ be the two pullbacks of $\mathcal{L}_{p}$ to $P \times P$, then $c_{1}\left(\mathcal{L}(2)_{p}\right)=c_{1}\left(\mathcal{L}_{1}\right)+c_{1}\left(\mathcal{L}_{2}\right)$ and $c_{2}\left(\mathcal{L}(2)_{p}\right)=c_{1}\left(\mathcal{L}_{1}\right) c_{1}\left(\mathcal{L}_{2}\right)$. Then:

$$
\begin{aligned}
\left\langle c_{1}^{m}\left(\mathcal{L}(2)_{p}\right) c_{2}^{n}\left(\mathcal{L}(2)_{p}\right) ; P(2)\right\rangle & =\left\langle\left(c_{1}\left(\mathcal{L}_{1}\right)+c_{1}\left(\mathcal{L}_{2}\right)\right)^{m} c_{1}\left(\mathcal{L}_{1}\right)^{n} c_{1}\left(\mathcal{L}_{2}\right)^{n} ; P \times P\right\rangle \\
& =\left(\begin{array}{c}
m \\
m / 2
\end{array}\right)\left\langle c_{1}^{k d / 2}\left(\mathcal{L}_{1}\right) c_{1}^{k d / 2}\left(\mathcal{L}_{2}\right) ; P \times P\right\rangle \\
& =\left(\begin{array}{c}
k d-2 n \\
k d / 2-n
\end{array}\right) k^{2},
\end{aligned}
$$

since $c_{1}^{k d / 2}\left(\mathcal{L}_{i}\right)=k$ by Theorem 2.9. This gives the first term in the calculation. For the second contribution, observe that if $L_{d / 2}$ is a universal line bundle on $C \times J_{d / 2}$, and $T P$ is the tangent bundle to $P$, then

$$
s_{t}\left(\nu\left(\Delta_{P}\right)\right)=s_{t}(T P)=s_{t}\left(\left(\rho_{*} L_{d / 2}\right)^{\oplus k}(1)\right)
$$

from the relative Euler sequence and the fact that $T C=\mathcal{O}_{C}$.

Since in addition, we have $\left.c_{1}(\mathcal{L}(2))\right|_{\Delta_{P}}=2 c_{1}(\mathcal{L})$ and $\left.c_{2}(\mathcal{L}(2))\right|_{\Delta_{P}}=c_{1}^{2}(\mathcal{L})$, we see that the second contribution is one-half the evaluation on $P$ of the $t^{m / 2}$ coefficient of

$$
-c_{t}\left(\oplus^{k}\left(\rho_{*} L_{d / 2}\right)(1)\right)^{-1}\left(1+2 c_{1}\left(\mathcal{L}_{p}\right) t\right)^{m}\left(c_{1}\left(\mathcal{L}_{p}\right)+c_{1}^{2}\left(\mathcal{L}_{p}\right) t\right)^{n}
$$

Let $a \in \mathbb{Z}$ be defined by $a[\Theta]=c_{1}\left(L_{d / 2}\right)_{p}$ and let $[z]=c_{1}\left(\mathcal{O}_{P}(1)\right)$. Then recall that $c_{1}\left(\mathcal{L}_{p}\right)=a[\Theta]+[z]$ and this is independent of the choice of $L_{d / 2}$. It follows from Grothendieck Riemann-Roch that $c_{1}\left(\oplus^{k}\left(\rho_{*} L_{d / 2}\right)\right)=k(a d / 2-1)[\Theta]$. The computations become more manageable if we make the following change of variables:

$$
w=z+(a-2 / d) \Theta, \quad \varepsilon=(2 / d) \Theta
$$

This change of variables is motivated by the observation that if there were a choice of universal bundle $L_{d / 2}$ such that $a=2 / d$, then $\rho_{*} L_{d / 2}$ would have the Chern classes of the trivial bundle, and $c_{t}\left(\left(\rho_{*} L_{d / 2}\right)^{\oplus k}(1)\right)$ would be simply $(1+z t)^{k d / 2}$. Such a universal bundle does not exist for $d>2$ since $2 / d$ is not an integer; nevertheless, via this change of variables we may pretend that $w$ represents $\mathcal{O}(1)$ for such a universal line bundle. In terms of the new variables, we have the following identities:

$$
c_{t}\left(\left(\rho_{*} L_{d / 2}\right)^{\oplus k}(1)\right)=(1+w t)^{k d / 2}, \quad \varepsilon^{2}=0, \quad w^{k d / 2}=0, \quad w^{k d / 2-1} \varepsilon=2 / d .
$$

Then we have

$$
\begin{aligned}
(5.18) & =-\frac{(1+2(w+\varepsilon) t)^{m}(w+\varepsilon)^{n}(1+(w+\varepsilon) t)^{n}}{(1+w t)^{k d / 2}} \\
& =-w^{n}\left(\frac{(1+2(w+\varepsilon) t)^{m}}{(1+w t)^{m / 2}}\right)-n \varepsilon w^{n-1}\left(\frac{(1+2 w t)^{m+1}}{(1+w t)^{m / 2+1}}\right) \\
& =-2 m \varepsilon w^{n} t\left(\frac{(1+2 w t)^{m-1}}{(1+w t)^{m / 2}}\right)-n \varepsilon w^{n-1}\left(\frac{(1+2 w t)^{m+1}}{(1+w t)^{m / 2+1}}\right) .
\end{aligned}
$$


From the identity: $(1+2 s t)^{2 r-1}(1+s t)^{-r}=2^{2 r-2} s^{r-1}+$ other powers of $t$, we see that the coefficient of $t^{m / 2}$ in (5.18) evaluates to $-2 m(2 / d) 2^{m-2}-n(2 / d) 2^{m}=$ $-k 2^{m}$ on $P$, which finishes the calculation.

The Flip Calculations. Recall the "flip" diagram of Theorem 3.44:

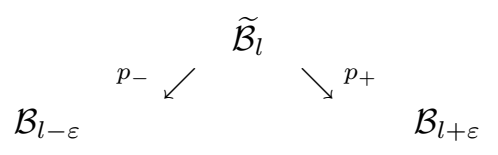

and its restriction to the exceptional divisor

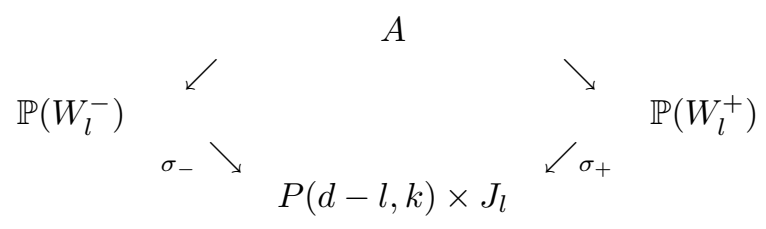

For the duration of the calculations it will be convenient to adopt the following conventions for naming various bundles:

(1) Denote by $U^{ \pm}$the universal rank two bundles on $C \times \mathcal{B}_{l \pm \varepsilon}$. Denote by $U_{p}^{ \pm}$the restriction of $U^{ \pm}$to $\{p\} \times \mathcal{B}_{l \pm \varepsilon}$, and finally denote by $c_{i}^{ \pm}$the pull-back of the $i$ th $(i=1,2)$ Chern class of $U_{p}^{ \pm}$to $\widetilde{\mathcal{B}}_{l}$.

Thus, according to this notation the flip contribution is the evaluation on the fundamental class of $\widetilde{\mathcal{B}}_{l}$ of the polynomial $\left(c_{1}^{-}\right)^{m}\left(c_{2}^{-}\right)^{n}-\left(c_{1}^{+}\right)^{m}\left(c_{2}^{+}\right)^{n}$.

(2) Denote by $L_{d}$ any choice of universal line bundle on $C \times J_{d}$. Denote by $L_{d}(x)$ the universal line bundle on the moduli space $P(d, k)$, so if $P(d, k)$ is identified with the projective bundle $\mathbb{P}\left(\left(\rho_{*} L_{d}\right)^{\oplus k}\right)$, then $\mathcal{O}(x)=\mathcal{O}_{P}(1)$.

(3) Denote by $\mathcal{O}_{\mathbb{P}\left(W_{l}^{-}\right)}(z)$ the line bundle $\mathcal{O}_{\mathbb{P}\left(W_{l}^{-}\right)}(1)$, so by Corollary 3.50, the normal bundle $\nu\left(\mathbb{P}\left(W_{l}^{-}\right)\right)=W_{l}^{+}(-z)$.

Throughout the computations, if $E$ is a vector bundle on a variety $Y$ and $f$ : $X \rightarrow Y$ is understood, then we will denote also by $E$ the pullback of $E$ to $Y$. We trust that the possible confusion arising from this is less than the confusion that would be caused by the proliferation of notation necessary to make everything precise. For example, we have already used $\mathcal{O}_{P}(1)$ to denote its pullback to $C \times P$ and $W_{l}^{+}$to denote its pullback to $\mathbb{P}\left(W_{l}^{-}\right)$.

Proposition 5.19. There is an exact sequence of sheaves on $C \times \widetilde{\mathcal{B}}_{l}$ :

$$
0 \rightarrow U^{-}(-A) \rightarrow U^{+} \rightarrow L_{d-l}(x) \rightarrow 0,
$$

where $L_{d-l}(x)$ is extended by zero from $C \times A$.

Proof. Let $\mathcal{K}$ be the kernel of the map $U^{+} \rightarrow L_{d-l}(x)$ obtained by pulling back the map in Proposition 3.51 (ii). The $k$ sections $\mathcal{O}^{k} \rightarrow U^{+}$pull back after twisting to give a map $\mathcal{O}^{k}(-A) \rightarrow \mathcal{K}$, hence $k$ sections of $\mathcal{K}(A)$. If this results in a family of $\tau-\varepsilon$ stable pairs, then the result follows immediately from the universal property of blowing up. But this is precisely the content of (3.14) of Thaddeus [T2] in the case where $k=1$, and the general case is the same.

The following global versions of (3.41) and (3.42) will be essential to the calculation of the Segre polynomial of $\nu\left(\mathbb{P}\left(W_{l}^{-}\right)\right)$: If $\rho: C \times P(d-l, k) \times J_{l} \rightarrow P(d-l, k) \times J_{l}$ 
is the projection, then by looking at (3.41) and the long exact sequence of the double complex associated to (3.42) we have:

(i) $W_{l}^{-}=R^{1} \rho_{*}\left(L_{l}^{*} \otimes L_{d-l}(x)\right)$ and

(ii) $0 \rightarrow \rho_{*}\left(L_{l} \otimes L_{d-l}^{*}(-x)\right) \rightarrow\left(\rho_{*} L_{l}\right)^{\oplus k} \rightarrow W_{l}^{+} \rightarrow R^{1} \rho_{*}\left(L_{l} \otimes L_{d-l}^{*}(-x)\right) \rightarrow 0$.

From now on, assume that $C$ is elliptic. Then the long exact sequence in (ii) reduces to a short exact sequence, since $R^{1} \rho_{*}\left(L_{l} \otimes L_{d-l}^{*}(-x)\right)=0$ if $2 l-d>0$. This also implies that the first term of (ii) is a vector bundle, of rank $2 l-d$, and we will need the following:

Lemma 5.20. The top Chern class $c_{2 l-d}\left(\rho_{*}\left(L_{l} \otimes L_{d-l}^{*}(-x)\right) \otimes \mathcal{O}_{\mathbb{P}\left(W_{l}^{-}\right)}(-z)\right)$ vanishes.

Proof. Consider the following general setup. Suppose $X$ is any smooth projective variety, $\mathcal{L}$ is a line bundle on $C \times X$, and $\rho: C \times X \rightarrow X$ is the projection. Let $E=\rho_{*} \mathcal{L}$ and let $F=R^{1} \rho_{*} \mathcal{L}^{*}$, and suppose that $R^{1} \rho_{*} \mathcal{L}$ and $\rho_{*} \mathcal{L}^{*}$ both vanish, so $E$ and $F$ are vector bundles on $X$, of the same rank $n$, since $C$ is assumed to be elliptic. Now, by Grothendieck-Riemann-Roch and the fact that the Todd class of an elliptic curve is trivial, it follows that the Chern classes of $E$ are the same as the Chern classes of $F^{*}$, so since the top Chern class $c_{n}\left(F^{*} \otimes \mathcal{O}_{\mathbb{P}(F)}(-1)\right)$ is easily seen to vanish, it follows that $c_{n}\left(E \otimes \mathcal{O}_{\mathbb{P}(F)}(-1)\right)$ vanishes as well. The lemma follows if we let $\mathcal{L}$ be $L_{l} \otimes L_{d-l}^{*}(-x)$.

Armed with 5.19 and 5.20, we can finally compute:

Calculation 5.21. If $C$ is elliptic and $l \in(d / 2, d)$, then we have:

$$
\left\langle c_{1}^{m} c_{2}^{n} ; \mathcal{B}_{l-\varepsilon}(d, 2, k)\right\rangle-\left\langle c_{1}^{m} c_{2}^{n} ; \mathcal{B}_{l+\varepsilon}(d, 2, k)\right\rangle=(-1)^{d} k^{2}\left(\begin{array}{c}
k d-2 n \\
k l-n
\end{array}\right)
$$

where by convention the right hand side is zero if $k l-n<0$ or $k l-n>k d-2 n$.

Proof. By Propositions 5.16 and 5.19, we see that the quantity we need to compute is the evaluation on $\mathbb{P}\left(W_{l}^{-}\right)$of the coefficient of $t^{\operatorname{dim} \mathbb{P}\left(W_{l}^{-}\right)-n}$ in the power series:

$$
-c_{t}\left(\nu\left(W_{l}^{+}(-z)\right)\right)^{-1}\left(1+c_{1}^{-} t\right)^{m}\left(c_{1}\left(L_{d-l}(x)\right)_{p}+c_{2}^{-} t\right)^{n} .
$$

From the exact sequence (ii) above, we have:

$$
c_{t}\left(\nu\left(W_{l}^{+}(-z)\right)\right)^{-1}=c_{t}\left(\oplus^{k}\left(\rho_{*} L_{l}\right)(-z)\right)^{-1} c_{t}\left(\rho_{*}\left(L_{l} \otimes L_{d-l}^{*}(-x)\right)(-z)\right)
$$

and from part (i) of Proposition 3.44, we see that when restricted to $\mathbb{P}\left(W_{l}^{-}\right)$, we have:

$$
\begin{aligned}
& c_{1}^{-}=c_{1}\left(L_{d-l}(x)\right)_{p}+c_{1}\left(L_{l}\right)_{p}-z, \\
& c_{2}^{-}=c_{1}\left(L_{d-l}(x)\right)_{p}\left(c_{1}\left(L_{l}\right)_{p}-z\right) .
\end{aligned}
$$

Suppose $c_{1}\left(L_{l}\right)_{p}=a\left[\Theta_{l}\right]$ and $c_{1}\left(L_{d-l}\right)_{p}=b\left[\Theta_{d-l}\right]$. Then as in Calculation 5.17, the following change of variables simplifies things considerably:

$$
\begin{aligned}
\varepsilon_{l} & =\frac{1}{l} \Theta_{l}, & \varepsilon_{d-l} & =\frac{1}{d-l} \Theta_{d-l}, \\
y & =x+b \Theta_{d-l}+\varepsilon_{l}, & w & =z-a \Theta_{l}+\varepsilon_{l},
\end{aligned}
$$


and while we are at it, let $E=\rho_{*}\left(L_{l} \otimes L_{d-l}^{*}(-x)\right)(-z)$.

In terms of these variables, the power series (5.22) becomes:

$$
\begin{gathered}
(5.22)=-(1-w t)^{-k l} c_{t}(E)(1-w t+y t)^{m}\left(y-\varepsilon_{l}\right)^{n}\left(1-w t+\varepsilon_{l} t\right)^{n} \\
=-c_{t}(E)\left[\sum_{p=0}^{m}\left(\begin{array}{c}
m \\
p
\end{array}\right)(y t)^{p}(1-w t)^{N-p}\left\{y^{n}+n \varepsilon_{l} t y^{n}(1-w t)^{-1}-n \varepsilon_{l} y^{n-1}\right\}\right],
\end{gathered}
$$

where $N=n+m-k l$, since $\varepsilon_{l}^{2}=0$. In addition, one checks that when evaluated on $P(d-l, k) \times J_{l}$, the following hold: $\varepsilon_{l} y^{k(d-l)}=k / l, y^{k(d-l)+1}=(k(d-l)+1) k / l$ and $y^{p}=0$ for $p>k(d-l)+1$. These, together with Lemma 5.20, imply that only three terms contribute to the coefficient of $t^{\operatorname{dim}\left(\mathbb{P}\left(W_{l}^{-}\right)\right)-n}$; namely:

$$
\begin{array}{r}
-c_{t}(E) t^{-n}\left\{\left(\begin{array}{c}
m \\
k(d-l)-n+1
\end{array}\right)\right. \\
{\left[\frac{(y t)^{k(d-l)+1}}{1-w t}-n \varepsilon_{l} t \frac{(y t)^{k(d-l)}}{1-w t}\right]} \\
\left.+\left(\begin{array}{c}
m \\
k(d-l)-n
\end{array}\right) n \varepsilon_{l} t \frac{(y t)^{k(d-l)}}{1-w t}\right\} .
\end{array}
$$

Now, since $c_{t}(E)$ behaves like $(1-w t)^{2 l-d}$ when paired with the classes $y^{k(d-l)+1}$ and $\varepsilon_{l} y^{k(d-l)}$, the calculation is reduced to finding the coefficient of $t^{2 l-d+k(d-l)-n}$ in the following polynomial:

$$
\begin{aligned}
-(1-w t)^{2 l-d-1} t^{k(d-l)+1-n}\{ & \left(\begin{array}{c}
m \\
k(d-l)-n+1
\end{array}\right)\left[y^{k(d-l)+1}-n \varepsilon_{l} y^{k(d-l)}\right] \\
& \left.+\left(\begin{array}{c}
m \\
k(d-l)-n
\end{array}\right) n \varepsilon_{l} y^{k(d-l)}\right\}
\end{aligned}
$$

which evaluates to

$$
\begin{gathered}
-(-1)^{2 l-d-1}\left\{\left(\begin{array}{c}
m \\
k(d-l)-n+1
\end{array}\right) \frac{(k(d-l)-n+1) k}{l}+\left(\begin{array}{c}
m \\
k(d-l)-n
\end{array}\right) \frac{n k}{l}\right\} \\
=(-1)^{d} k^{2}\left(\begin{array}{c}
k d-2 n \\
k l-n
\end{array}\right)
\end{gathered}
$$

as desired.

Finally, we use calculations 5.14, 5.17 and 5.21 to compute the Gromov invariants associated to maps from an elliptic curve $C$ to $G(2, k)$. Suppose $d$ is a non-negative integer. Then the expected dimension of $\overline{\mathcal{M}}_{Q}(d, 2, k)$ is $d k$, and thus by Theorem 5.5 , the intersection pairing $\left\langle X_{1}^{m} X_{2}^{n}\right\rangle$ for $m+2 n=k d$ is realized as $\left\langle c_{1}^{m} c_{2}^{n+k \delta}\right.$; $\left.\mathcal{B}_{\tau}(d+2 \delta)\right\rangle$ for sufficiently large $\delta$. Also, $\tau$, following the proof of Theorem 4.24, may be chosen to be $d+\delta+\varepsilon$.

This pairing on $\mathcal{B}_{\tau}(d+2 \delta)$ is now computed by first computing the corresponding pairing on $\mathcal{B}_{d / 2+\varepsilon}$, which is either $k 2^{m-1}$ if $d$ is odd, by Calculation 5.14 , or $-k 2^{m-1}+\frac{k^{2}}{2}\left(\begin{array}{c}m \\ m / 2\end{array}\right)$ if $d$ is even, by Calculation 5.17. The correction terms, which 
measure how the pairing changes as $\tau$ moves from $d / 2+\delta+\varepsilon$ to $d+\delta+\varepsilon$, are in both the even and odd case equal to

$$
(-1)^{d} k^{2} \sum_{l=[d / 2+1]+\delta}^{d+\delta}\left(\begin{array}{c}
m \\
k l-(n+k \delta)
\end{array}\right)=(-1)^{d} k^{2} \sum_{l=[d / 2+1]}^{d}\left(\begin{array}{c}
m \\
k l-n
\end{array}\right)
$$

by repeated application of Calculation 5.21. If we combine these results, we finally get:

$$
\left\langle X_{1}^{k d-2 n} X_{2}^{n}\right\rangle=(-1)^{d+1} k 2^{k d-2 n-1}-(-1)^{d+1} \frac{k^{2}}{2} \sum_{\substack{p \in \mathbb{Z} \\
n / k \leq p \leq d-n / k}}\left(\begin{array}{c}
k d-2 n \\
k p-n
\end{array}\right)
$$

which proves Theorem 1.7. Thus, comparing with (5.13), the conjecture of Vafa and Intriligator is true in these cases as well:

Theorem 5.23. Conjecture 5.10 is true for maps from an elliptic curve to $G(2, k)$.

\section{ACKNOWLEDGEMENT}

We thank the referee for carefully reading the manuscript and for pointing out an incorrect proof in an earlier version.

\section{REFERENCES}

[A-B] Atiyah, M. F. and R. Bott, The Yang-Mills equations over Riemann surfaces, Phil. Trans. R. Soc. Lond. A 308 (1983), 523-615. MR 85k:14006

[A-C-G-H] Arbarello, E., M. Cornalba, P. Griffiths, and J. Harris, Geometry of Algebraic Curves, Vol. I, Springer-Verlag, New York, Berlin, Heidelberg, 1985. MR 86h:14019

[B] Bradlow, S. B., Special metrics and stability for holomorphic bundles with global sections, J. Diff. Geom. 33 (1991), 169-214. MR 91m:32031

[B-D1] Bradlow, S. B. and G. D. Daskalopoulos, Moduli of stable pairs for holomorphic bundles over Riemann surfaces, Int. J. Math. 2 (1991), 477-513. MR 93b:58026

[B-D2] Bradlow, S. B. and G. D. Daskalopoulos, Moduli of stable pairs for holomorphic bundles over Riemann surfaces II, Int. J. Math. 4 (1993), no. No. 6, 903-925. CMP 94:05

[B-D-W] Bradlow, S., G. Daskalopoulos, and R. Wentworth, Birational equivalences of vortex moduli, Topology (to appear).

[B-T] Bott, R. and L. Tu, Differential Forms in Algebraic Topology, Springer-Verlag, New York, Berlin, Heidelberg, 1982. MR 83i:57016

[Be] Bertram, A., Moduli of rank 2 vector bundles, theta divisors, and the geometry of curves on projective space, J. Diff. Geom. 35 (1992), 429-469. MR 93g:14037

[Br] Bruguières, A., The scheme of morphisms from an elliptic curve to a Grassmannian, Comp. Math. 63 (1987), 15-40. MR 88g:14009

[D-U] Daskalopoulos, G. and K. Uhlenbeck, An application of transversality to the topology of the moduli space of stable bundles, Topology 34 (1994), 203-215.

[F] Floer, A., Symplectic fixed points and holomorphic spheres, Commun. Math. Phys. 120 (1989), 575-611. MR 90e:58047

[Ful] Fulton, W., Intersection Theory, Springer-Verlag, New York, Berlin, Heidelberg, 1984. MR 85k:14004

[G1] Gromov, M., Pseudo holomorphic curves in symplectic manifolds, Invent. Math. 82 (1985), 307-347. MR 87j:53053

[G2] Gromov, M., Soft and hard symplectic geometry, in Proceedings of the International Congress of Mathematicians, Berkeley, 1986. MR 91c:53034 
[G-H] Griffiths, P. and J. Harris, Principles of Algebraic Geometry, Wiley, New York, 1978. MR 80b:14001

[G-S] Guillemin, V. and S. Sternberg, Birational equivalence in the symplectic category, Invent. Math. 97 (1989), 485-522. MR 90f:58060

[Gro] Grothendieck, A., Techniques de construction et théorèmes d'existence en géometrie algébrique IV: Les schémas de Hilbert, Séminaire Bourbaki 221 (1960/61). MR 27:1339

[I] Intriligator, K., Fusion residues, Mod. Phys. Lett. A6 (1991), 3543-3556. MR 92k: 81180

[K2] Kirwan, F., On the homology of compactifications of moduli spaces of vector bundles over a Riemann surface, Proc. London Math. Soc. (3) 53 (1986), 237-266. MR 88e: 14012

[Ko] Kobayashi, S., Differential Geometry of Complex Vector Bundles, Princeton University Press, 1987. MR 89e:53100

[N] Newstead, P.E., Introduction to Moduli Problems and Orbit Spaces, Tata Inst. Lectures 51 (1978), Springer-Verlag, Heidelberg. MR 81k: 14002

[R] Ruan, Y., Toplogical sigma model and Donaldson type invariants in Gromov theory, preprint, 1993.

[S-U] Sacks, J. and K. K. Uhlenbeck, The existence of minimal immersions of 2-spheres, Ann. Math. 113 (1981), 1-24. MR 82f:58035

[Str] Strømme, S., On parameterized rational curves in Grassmann varieties, in Lecture Notes in Math. 1266, Springer-Verlag, New York, Berlin, Heidelberg, 1987. MR 88i:14020

[T1] Thaddeus, M., Conformal field theory and the cohomology of the moduli space of stable bundles, J. Diff. Geom. 35 (1992), 131-149. MR 93g:14017

[T2] Thaddeus, M., Stable pairs, linear systems, and the Verlinde formula, Invent. Math. 117 (1994), 317-353. MR 95e:14006

[Ti] Tiwari, S., preprint.

[V] Vafa, C., Topological mirrors and quantum rings, in "Essays on Mirror Manifolds" S.-T. Yau, ed., International Press, Hong Kong, 1992. MR 94c:81193

[Wf] Wolfson, J., Gromov's compactness of pseudo-holomorphic curves and symplectic geometry, J. Diff. Geom. 28 (1988), 383-405. MR 89m:53058

[Wi] Witten, E., Topological sigma models, Commun. Math. Phys. 118 (1988), 411-449. MR 90b: 81080

[Z] Zagier, D., unpublished.

Abstract. Two compactifications of the space of holomorphic maps of fixed degree from a compact Riemann surface to a Grassmannian are studied. It is shown that the Uhlenbeck compactification has the structure of a projective variety and is dominated by the algebraic compactification coming from the Grothendieck Quot Scheme. The latter may be embedded into the moduli space of solutions to a generalized version of the vortex equations studied by Bradlow. This gives an effective way of computing certain intersection numbers (known as "Gromov invariants") on the space of holomorphic maps into Grassmannians. We carry out these computations in the case where the Riemann surface has genus one.

Department of Mathematics, University of Utah, Salt Lake City, Utah 84112

E-mail address: bertram@math.utah.edu

Department of Mathematics, Brown University, Providence, Rhode Island 02912

E-mail address: daskal@gauss.math.brown.edu

Department of Mathematics, University of California, Irvine, California 92717

E-mail address: raw@math.uci.edu 